\title{
Globular Clusters in 19 Northern Abell Clusters ${ }^{1}$
}

\author{
John P. Blakeslee ${ }^{2}$, John L. Tonry ${ }^{3}$, AND Mark R. Metzger ${ }^{2}$ \\ Dept. of Physics, 6-216, Massachusetts Institute of Technology, Cambridge, MA 02139
}

\begin{abstract}
We use the method developed by Blakeslee \& Tonry (1995) to study the globular cluster (GC) populations of 21 giant elliptical galaxies in 19 Abell clusters. This method, applied here primarily in the $R$ band, is based on the surface brightness fluctuations technique of extragalactic distance measurement. The sample galaxies range in redshift from 5000 to $10,000 \mathrm{~km} \mathrm{~s}^{-1}$, and were selected primarily from the Lauer \& Postman (1994) survey of brightest cluster galaxies (BCGs).

We find a tight correlation between the GC specific frequency $S_{N}$ of the central bright galaxy in the cluster and the cluster velocity dispersion. $S_{N}$ also correlates well with the cluster X-ray temperature and with the number of bright neighboring galaxies, less well with the galaxy profile, and only marginally with galaxy luminosity and overall cluster richness. It does not correlate with cluster morphology class. Thus, unlike galaxy luminosity, $S_{N}$ is determined by the cluster mass, or density. To account for this situation, we propose that the GCs formed early and in proportion to the available mass, while the luminosity growth of the galaxy was later halted, yielding the observed correlations of $S_{N}$ with density. We introduce a quantity called $\eta_{G C}$, the number of GCs per unit local cluster mass. For a simple cluster mass model, $\eta_{G C}$ is found to be constant, indicating a uniform GC production rate per unit available mass.

A measurement of the Gaussian width $\sigma$ of the GC luminosity function (GCLF) is one of the byproducts of our analysis. In the cosmic microwave background frame, the mean width for this sample is $\langle\sigma\rangle=1.43 \mathrm{mag}$, virtually identical to the HST value for M87, the galaxy used to calibrate the mean of the GCLF in this analysis.
\end{abstract}

Subject headings: galaxies: clusters: general — galaxies: distances and redshifts galaxies: elliptical and lenticular, cD - galaxies: star clusters - globular clusters: general

\footnotetext{
${ }^{1}$ Observations conducted at the Michigan-Dartmouth-MIT Observatory

${ }^{2}$ Current address: Palomar Observatory, California Institute of Technology, Mail Stop 105-24, Pasadena, CA 91125; jpb@astro.caltech.edu, mrm@astro.caltech.edu

${ }^{3}$ Current address: Institute for Astronomy, University of Hawaii, 2680 Woodlawn Drive, Honolulu, HA 96822; jt@avidya.ifa.hawaii.edu
} 


\section{INTRODUCTION}

The first reported observations of a globular cluster system (GCS), or population, around an external galaxy was by Hubble (1932), who "provisionally identified as globular clusters" the relatively bright, slightly extended objects in the halo of M31. Progress in the study of extragalactic GCs was slow however, and it was more than twenty years before Baum (1955) identified the brightest members of the extremely rich GCS which surrounds M87, the central giant elliptical in the Virgo cluster. More detailed photographic studies of this system would be another decade in coming (Racine 1968a,b).

The first GCS to be observed at a distance significantly beyond Virgo was by Smith \& Weedman (1976), who reported a statistical excess of $\sim 20$ stellar objects around NGC 3311, the cD galaxy at the center of the Hydra cluster. At $\sim 3500 \mathrm{~km} \mathrm{~s}^{-1}$, Hydra is one of the nearest Abell clusters (Abell 1060), and the detection by Smith \& Weedman represented $\$ 0.2 \%$ of the total GC population of this galaxy. Significant further progress in the study of GCSs, particularly around M87-like galaxies in clusters, awaited advances in astronomical imaging and analysis techniques.

The revolution in astronomy sparked by CCDs reached the field of extragalactic GC research in the mid-1980s. Van den Bergh et al. (1985) used the new tool to study the M87 GCS, reaching for the first time beyond the turnover point in this galaxy's globular cluster luminosity function (GCLF). CCD studies of GCs around ellipticals in Leo (Pritchet \& van den Bergh 1985), Coma (Harris 1987; Thompson \& Valdes 1987), and Virgo (Cohen 1988) followed soon after. With the revolutionary image quality of the Hubble Space Telescope (HST), it is now possible to image two magnitudes beyond the turnover in the M87 GCLF, (Whitmore et al. 1995), reach unprecedented depths along the Coma GCLF (Baum et al. 1995), and study a larger number of smaller GCSs in detail (Forbes et al. 1996a).

Besides the boon to GCS research, CCDs allowed for the development of the SBF method of distance measurement (Tonry \& Schneider 1988; but see also Shopbell et al. 1993, who showed that it could be done, with considerable effort, on photographic plates). The SBF method measures the seeing-convolved variance, or "fluctuations," produced by the Poisson statistics of the stars in an early-type galaxy. The amplitude of the fluctuations decreases with the square of the distance to the galaxy, and when divided by the galaxy's mean surface brightness, yields the luminosity-weighted average flux of the stars within the galaxy. This flux, usually referred to in terms of magnitudes and called $\bar{m}$, gives the distance to the galaxy after proper calibration.

In the present work, the SBF image analysis methods are used to measure the "bumpiness" in a galaxy image due to GCs. Traditionally, GC populations around galaxies have been studied through their brightest members, which appear as an excess of faint point sources with roughly the same distribution as the halo light. However, they can also be studied through the surface brightness variance ("bumpiness") produced in the image by the remainder of the population (those too faint to be detected as point sources). This variance is a nuisance which must be subtracted from the stellar SBF amplitude in order to derive a distance, as in the SBF survey 
(Tonry et al. 1997). However, it can also be used as a probe of the GC population. By using the counts of the brightest GCs together with the variance from the rest of the GCs, we can constrain the luminosity function and determine the total GC population much more accurately, and to much larger distances.

The number of GCs per unit $M_{V}=-15$ of galaxy luminosity is known as the "specific frequency (or number) of globular clusters," abbreviated $S_{N}$ (Harris \& van den Bergh 1981). Some luminous central galaxies in clusters, M87 being the most famous, have huge GC populations with $S_{N} \sim 12$, about three times the typical value for giant ellipticals. Speculation on the origin of these "anomalous", or "high- $S_{N}$ ", systems has abounded, but there have been few observational constraints. In the first application of our analysis method (Blakeslee \& Tonry 1995; hereafter BT95), we studied the GC populations of the two central giants in the Coma cluster, nearly six times more distant than Virgo. Due to the success of that project, we undertook a larger, systematic study of an unbiased sample of brightest galaxies in Abell clusters using the same technique. Our goal was to make significant progress towards understanding the seemingly unpredictable variations in the observed $S_{N}$ values of these galaxies. Here we report on the results of that study.

Along the way, we have learned more about the GCLF, usually represented by a Gaussian distribution in magnitudes:

$$
N(m)=\left(2 \pi \sigma^{2}\right)^{-\frac{1}{2}} N_{0} e^{\frac{-\left(m-m^{0}\right)}{2 \sigma^{2}}},
$$

where $N_{0}$ is the total number density of GCs, $m^{0}$ is the apparent mean, or "turnover" magnitude, and $\sigma$ is the Gaussian dispersion, or "width". The GCLF has been used extensively as a distance indicator based upon the universality of the absolute magnitude of the turnover $M^{0}$ (see Whitmore 1996 and references therein). HST increases by at least a factor of five the maximum distance to which this method can be used and may soon resolve the lingering questions regarding possible variations in $M^{0}$. In our initial Coma study, we extended by a factor of five the distance to which GCLF widths had been measured, and subsequent work reported here has gone further still.

In the following section we discuss the sample selection for this study and describe the observations. In Section 3, we detail the data reduction and analysis procedure, essentially the same as that of BT95. Section 4 presents the results on the specific frequencies and GCLFs of the sample galaxies, and Section 5 explores the relationship of $S_{N}$ to other properties of the galaxies and the galaxy clusters. We find the first clear evidence for strong correlations of $S_{N}$ with cluster properties; some of which were presented previously by Blakeslee (1997a). In Section 6, we compare our results to those predicted by various models and attempt to synthesize the simplest scenario consistent with observations. The final section summarizes this work. 


\section{SAMPLE SELECTION AND OBSERVATIONS}

\subsection{Abell Cluster Galaxy Sample}

The galaxy sample used in this study was selected from the Lauer \& Postman (1994; hereafter LP) volume-limited survey of brightest cluster galaxies (BCGs) in 119 Abell and ACO galaxy clusters (Abell 1958; Abell, Corwin, \& Olowin 1989). The LP sample includes nearly all the Abell/ACO clusters with redshift $c z<15,000 \mathrm{~km} \mathrm{~s}^{-1}$ and galactic latitude $|b|>15^{\circ}$. Seven clusters were excluded from their survey because of spiral, star-forming irregular, or anomalously faint BCGs, six "clusters" were not true galaxy over-densities, and two were not observed by LP. Postman \& Lauer (1995; hereafter PL) present and discuss the data used by LP, including observations of second brightest, or second ranked, galaxies in clusters for which the magnitude offset between first and second was small.

To ensure that reasonably accurate statistical statements about GCSs would result, we selected as complete a subsample as possible from the LP survey. The final sample studied here includes all of the northern hemisphere LP and PL galaxies with $c z<10,000 \mathrm{~km} \mathrm{~s}^{-1}$ and $\alpha>0.4$, where $\alpha \equiv d \log L /\left.d \log r\right|_{r_{m}}$ is the logarithmic slope of the galaxy luminosity evaluated at the metric radius $r_{m}=10 h^{-1} \mathrm{kpc}$. (Larger $\alpha$ implies a more extended galaxy; it was first introduced by Hoessel [1980], who called it the "structure parameter" of the galaxy profile). In addition, NGC 4839 in Coma has been added to this sample because it is a known cD galaxy, though not the first or second brightest Coma member. ( $\mathrm{A}$ cD galaxy is a giant elliptical that possesses an extended, low surface brightness halo; its radial profile exhibits a significant excess of light relative to an $r^{1 / 4}$ law. See Tonry [1987] for a review.) Thus, the sample comprises 23 galaxies in 19 Abell clusters, two of which are the Coma galaxies studied by BT95. Of the nine true clusters omitted by LP, only A426 (the Perseus cluster, with its giant disturbed BCG Perseus A) and A400 (which LP reject as "anomalously faint") are within the above redshift and coordinate cutoffs.

Table 1 lists the galaxies in the present sample. The columns are: galaxy identification (Abell number of, and galaxy rank in, cluster); right ascension and declination (J2000 coordinates); galactic longitude and latitude; heliocentric velocity in $\mathrm{km} \mathrm{s}^{-1} ; B$-band extinction (as listed by Burstein \& Heiles 1984); $R$-band absolute metric magnitude and $\alpha$ parameter (both as listed in LP and PL, except A1656-3, for which the values were determined from the data presented here); and the "common name" for each galaxy. The common galaxy name was chosen first to be the NGC or IC number (prefixed by N or I, respectively), then the UGC number (Nilson 1973; prefixed by $\mathrm{U}$ ), and last by the CGCG number (Zwicky 1968; prefixed by C). The table also notes the galaxies found by Schombert (1988) to possess cD envelopes.

Table 2 contains reference information on the galaxy clusters themselves. For each cluster, the table lists J2000 coordinates of the centroid of the extended X-ray emission (Jones \& Forman 1997); mean velocities in the heliocentric, cosmic microwave background (CMB), and "Abell cluster inertial" (ACI) frames $\left(\mathrm{km} \mathrm{s}^{-1}\right.$; from PL and Postman 1996); velocity dispersion of the 
member galaxies $\left(\mathrm{km} \mathrm{s}^{-1}\right.$; described in $\left.\$ 5.2\right)$; X-ray gas temperature $(\mathrm{keV}$; from Jones \& Forman 1997); richness class and Bautz-Morgan type (Abell et al. 1989); and Rood-Sastry type (Struble \& Rood 1987). The ACI frame is the frame in which LP determined their clusters to have no net peculiar velocity according to the $L_{m}-\alpha$ distance indicator. The only cluster in the sample with a colloquial name is Abell 1656, the Coma cluster.

\subsection{Observing Procedure and Runs}

The data used in this study were obtained with the $2.4 \mathrm{~m}$ telescope at the MichiganDartmouth-MIT (MDM) Observatory on Kitt Peak over the course of several observing runs. The integration times for the individual exposures on the program galaxies ranged from 10 to 15 minutes. Between exposures, the telescope was shifted by $5-10^{\prime \prime}$ in order to improve the image flattening, allow for the removal of cosmic ray hits and CCD defects, and provide a check on whether a series of exposures was truly photometric. The total integration times on the program galaxies ranged from just over $1 \mathrm{hr}$ to nearly $6.5 \mathrm{hr}$, determined mainly by the galaxy's distance. Each night of observing, approximately 10 twilight flat fields were taken through the filter which was to be used for the deep imaging (either $R$ or $I$ ). If the sky was clear, about 10 images of Landolt (1992) photometric standard star fields were taken at varying airmasses throughout the night in each band for which photometry was needed. Standard star reductions and photometric calibration of the final galaxy images are discussed in $\$ 3.1$.

Table 3 summarizes the information on all the observing runs. The runs are designated by the month and year in which they took place. For each run, the table lists the CCD camera that was used, the pixel scale of the images in arcseconds per pixel, the filter through which the GC data were taken, the typical photometric coefficients $\left(m_{1}, A\right.$, and $C$, defined below in Eq. [2]) used in calibrating the GC data, and some notes. (The CCD listed as "STIS 2048" was fabricated as part of the STIS program and obtained by MDM, although the STIS chip later installed on HST is a $1024^{2}$ device.) Collecting data for this project was not the primary goal of either of the first two runs. Only the two Coma galaxies from BT95 were observed in the 0593 run, and only one sample galaxy was observed during 0794 . The 0593 images were binned $2 \times 2$ to yield $0^{\prime \prime} 343 \mathrm{pix}^{-1}$. After imaging in the $I$ band for the first two runs, we chose to take the remainder of the data in $R$ for several reasons: (1) The $R$ band is near the peak sensitivity of the CCD, (2) thinned chips like the MDM Tek $1024^{2}$ (the detector of choice for its high quantum efficiency) show significant fringing in $I$, (3) the stellar SBF is relatively stronger in $I$, with $\bar{m}_{R}-\bar{m}_{I} \sim 1.6$, while $(R-I) \sim 0.5$ for GCs, (4) the sky is much brighter in $I$ than $R$, and (5) PL report precise aperture photometry in $R$, which can be used as a cross-check for our photometry (Blakeslee et al. 1998). A glass $R$ filter provides a much better match to the standard bandpass, so it was used in lieu of an interference filter; the reverse is true in the $I$ band, where the open red end of glass filters makes them a poor choice for CCD photometry. 


\section{REDUCTIONS AND ANALYSIS}

\subsection{Image Processing and Calibration}

High signal-to-noise flat fields were produced for each night as follows. The individual frames were bias corrected by fitting the overscan region of the CCD (which had variable top-to-bottom structure in the bias) to a $\sim 5$ th order spline and subtracting. Each frame was then windowed and divided by the straight sum of all the frames, allowing any stars present to be easily identified and masked out. The sum was then multiplied back in and deviant pixels due to cosmic rays hits were identified by an automatic procedure (Tonry et al. 1997). The pixels affected by the cosmic rays and stars were then replaced by scaled data values determined according to the pixels' values in the rest of the image stack. The frames were then summed to to produce the final flat field. The galaxy data and standard star calibration frames were bias subtracted and windowed in the same manner as the flats and divided by the normalized flat field.

The Landolt standard star fields used in this project are mostly the same as those listed by Tonry et al. (1997). They were selected because of the large number of observations made by Landolt on each, and consequent small magnitude uncertainties, and because of the number of stars present within a $\sim 5^{\prime} \times 5^{\prime}$ field. Following Landolt (1992), and as in Tonry et al. (1997), we summed the flux from each photometric standard within an aperture of $7^{\prime \prime}$ radius. Standard star measurements with estimated errors larger than 0.02 mag from flux, sky, and Landolt magnitude uncertainties were discarded. After all the standard star observations from a run were reduced, the results were fitted according to

$$
m=-2.5 \log (f / t)-A \sec (z)+C(V-I)+m_{1}
$$

where $m$ is the magnitude reported by Landolt in the appropriate band, $f$ the total counts in electrons from the star within $7^{\prime \prime}, t$ the exposure time in seconds, $z$ the zenith angle, and $(V-I)$ comes from Landolt. Typical values of $A, C$, and $m_{1}$ were given in Table 3 for each run. On truly clear nights, the scatter about the derived relation was $\lesssim 0.01$ mag.

The individual exposures making up a galaxy observation were bias subtracted and flattened before being brought into registration based on the positions of stars in the images. Bad pixels were masked, and the frames were then combined, rejecting cosmic ray hits, to make the final galaxy image. The following two subsections discuss the properties of the images which make up the present data set.

\subsection{Image "Quality"}

One measure of the depth of an observation is the quantity $m_{1}^{*}$, the magnitude of an object which will produce one count per total image integration time, corrected for Galactic extinction:

$$
m_{1}^{*}=2.5 \log (t)-A \sec (z)+C(V-I)+m_{1}-A_{\lambda},
$$


where $A_{\lambda}$ is the extinction in the $\lambda$ band. Thus, the extinction corrected magnitude of an object yielding $f$ total counts in the image can be calculated as: $m=-2.5 \log (f)+m_{1}^{*}$. So, $m_{1}^{*}$ indicates the amount of signal in an image, but takes no account of the noise.

It would be helpful to have some measure of the quality of an image, i.e., the suitability of an observation for yielding the desired information. The approximate signal-to-noise ratio (SNR) of a sky-limited object producing $f$ counts within the PSF full width at half maximum (FWHM) is

$$
\mathrm{SNR}=\frac{f}{\sqrt{b \times \mathrm{PSF}^{2}}}
$$

where $b$ is the sky level in counts/arcsec ${ }^{2}$ and PSF is the FWHM in arcsec. (To calculate the true signal-to-noise, the galaxy background would also need to be taken into account.) For a given luminosity, such as a fixed point along the GCLF, the flux received by the detector scales as $f \sim t / d^{2}$, where $d$ is the distance to the source. Thus, in order to reach the same SNR at this fixed luminosity, the exposure time must scale as $t \sim d^{4}$. Exposure times for our sample galaxies were scaled in this way (taking into account sky background and seeing variations), with the goal being to reach within $\sim 2$ mag or so of the expected GCLF turnover $m^{0}$ while the point source identification was still $\gtrsim 90 \%$ complete.

It is interesting to view Eq. (四) in terms of magnitudes:

$$
2.5 \log (\mathrm{SNR})=-2.5 \log (\mathrm{PSF})-m_{\mathrm{FW}}+0.5\left(m_{1}^{*}+\mu_{s k y}\right),
$$

where $m_{\mathrm{FW}}$ is the magnitude of the object within the PSF FWHM and $\mu_{s k y}$ is the sky surface brightness in mag/arcsec ${ }^{2}$. For a PSF which approximates a two dimensional Gaussian, the total flux is about twice the flux within the FWHM. The obvious characteristic magnitude for GC studies is $m^{0}$, the point at which the GCLF turns over. The limiting magnitudes of the images in this project do not generally approach $m^{0}$; however, if we plug in $m_{\mathrm{FW}}=m^{0}$, the object under consideration will be one which is twice as bright as $m^{0}$, e.g., a globular cluster 0.75 mag brighter than the GCLF turnover. Eq. (5) then becomes:

$$
2.5 \log \left(\mathrm{SNR}^{0}\right)=-2.5 \log (\mathrm{PSF})+0.5\left(m_{1}^{*}+\mu_{s k y}\right)-m^{0},
$$

where we have called this fiducial signal-to-noise ratio $\mathrm{SNR}^{0}$, although it actually refers to an object twice as bright as, not equal to, $m^{0}$. In $1^{\prime \prime}$ seeing, the quantity calculated in Eq. (6) is positive if the average of $m_{1}^{*}$ and $\mu_{s k y}$ is fainter than the GCLF turnover $m^{0}$ in the galaxy. Perhaps more intuitive, however, is $\mathrm{SNR}^{0}$ itself:

$$
\mathrm{SNR}^{0}=\mathrm{PSF}^{-1} \times 10^{0.2\left(m_{1}^{*}+\mu_{s k y}-2 m^{0}\right)} .
$$

This is a useful indicator of the data image quality once $m^{0}$ has been estimated.

We can estimate $m^{0}$ from its measured value in the Virgo cluster, first placing Virgo into the CMB frame using the relative distance between it and one of the sample clusters. From the review 
by van den Bergh (1992), we adopt $\Delta(m-M)_{C V}=3.71 \pm 0.10$ mag as the relative Coma-Virgo distance modulus. Recently, Baum et al. (1997) have found $\Delta(m-M)_{C V}=4.10 \pm 0.06 \mathrm{mag}$ from a maximum likelihood comparison of HST observations of the GCLF in IC 4051 with the HST GCLF from Whitmore et al. (1995). They also cite the work of Giovanelli (1996) as indicating $\Delta(m-M)_{C V}=3.95$ mag. However, the large, homogeneous "Mark III" Catalog (Willick et al. 1997) of Tully-Fisher distances yields $\Delta(m-M)_{C V}=3.66 \pm 0.15$ mag (Willick 1997), and a re-investigation of the $D_{n}-\sigma$ and fundamental plane relations in the two clusters finds $\Delta(m-M)_{C V}=3.55 \pm 0.15 \mathrm{mag}$ (D'Onofrio et al. 1997). The value we have adopted is in agreement with these determinations, while values near 4.0 mag are not. Thus, using the CMB velocity of Coma in Table 2, we have:

$$
m^{0}(z)=m_{\mathrm{Vir}}^{0}+5 \log \left(\frac{c z}{1310}\right) .
$$

This calibration is discussed further in $\S 3.6$.

\subsection{Final Data Set}

Table 4 presents the data set used for this study of the GCSs of Abell cluster galaxies. For each galaxy image, the table lists the observing run in which the data were taken, the total exposure time in seconds, the seeing FWHM in arcsec, $m_{1}^{*}$ as defined in Eq. (3), the sky brightness in $\mathrm{mag} / \operatorname{arcsec}^{2}, \mathrm{SNR}^{0}$ calculated as described above, and the total number of unique objects identified in the image down to a signal-to-noise limit of 4.0 by the photometry program DoPhot (Schechter, Mateo, \& Saha 1993; DoPhot and the point source photometry will be discussed in \$.4. Galaxies A347-1 and A569-1 were both observed twice, and each observation was reduced independently. For these two galaxies the strong dependence of the number of identified objects on $\mathrm{SNR}^{0}$ is clearly evident.

Of course, $\mathrm{SNR}^{0}$ does not tell the whole story. The total signal-to-noise of the data scales also with the square root of the number of GCs present in the image. This cannot be known ahead of time, though some estimates might be made based on galaxy luminosity. For comparison purposes, Table 1 includes the information on the Coma observations presented and analyzed by BT95. Both of these galaxies happened to have very rich GC populations. Consequently, it

was possible to learn a good deal about them, despite the fact that their $\mathrm{SNR}^{0}$ values are among the lowest in the table. (Although the comparison is not really fair, as those images were in the $I$-band where the sky is much brighter but the galaxy background is not, and we have neglected the galaxy background here.)

\subsection{Point Source Reductions}




\subsubsection{Producing a Smooth Background}

As in BT95, we mask out all the easily visible stars and small galaxies before fitting a smooth model to the galaxy light. For many of these fields, the main galaxy and several smaller galaxies were modeled and subtracted using an iterative procedure. In some of the images, however, the multiple galaxies are comparable in size, close together, and show interaction. For these galaxies, a single smooth galaxy model could not be fitted at the first iteration; thus, we used the simultaneous nucleus fitting software developed by Lauer (1988) and also used by LP. After simultaneously modeling and subtracting the primary galaxy and its secondary nuclei, we added the primary back in and could then successfully model it with our software. The same was done for the rest of the nuclei until all of them had been modeled and subtracted, minimizing the model residuals. An extreme case can be seen in Figure 1, which shows the model generated in this way for A539-2.

After subtracting the final galaxy model, we mask all visible objects and fit the large scale model residuals to a grid of spacing roughly 10 times the size of the PSF, interpolating between grid points. Subtraction of the large scale residuals leaves a very flat "residual image" on which the point source photometry and power spectrum measurements described below are carried out.

\subsubsection{Finding the Objects}

The automatic photometry program DoPhot (Schechter et al. 1993) was run on an integer version of each residual image in which saturated stars, "large" dwarf galaxies, and other bad features had been masked. Afterwards, DoPhot's model image (generated by taking the difference of the input and output images) was closely inspected for extended objects which had been fitted as tight bunches or strings of point sources. Objects larger than DoPhot's fit box cannot be fitted as single objects, but even some smaller ones refuse to conform to any reasonable model DoPhot might try. Thus, in all cases, it was necessary to mask out these problematic objects (which sometimes included residuals from the galaxy subtraction) and re-run DoPhot. Aside from these aberrant cases, DoPhot does a good job of distinguishing between point sources and extended objects, as tests performed by Ajhar, Blakeslee, \& Tonry (1994) showed. This proves to be useful for rejecting possible dwarf galaxies, perhaps as faint as the brightest GCs, which may cluster around the BCG.

DoPhot's fit magnitudes must be calibrated onto an absolute scale. This is done by taking the median difference of the aperture and fit magnitudes for the $\lesssim 20$ brightest objects. Judging from the scatter among these objects, the uncertainty in this correction is $0.02-0.03$ mag, or about twice as large as the photometric uncertainty from the standard star calibration. The total number of unique objects identified and fitted by DoPhot in each field were listed in Table 4 . These numbers do not precisely reflect the relative densities of objects among the images because the final field size varies due to unequal telescope shifting, windowing, and masking (as well as 
different detectors in a few cases), but they convey a general impression of the number of objects present in each residual image.

\subsubsection{Completeness}

In order to determine the completeness of DoPhot in finding point sources as a function of magnitude, a bright, but not saturated, isolated star was chosen from each field and cloned into a grid of stars with separation $\sim 10^{\prime \prime}$. The grid was then scaled $5-7$ times in 0.5 mag steps, with the noise in the grid increased appropriately for each scaling. The brightest scaling was at $m=22.0-23.0$, depending on the depth of the image. Each scaled grid of stars was added to a separate copy of the real data, and DoPhot was run on the image; all parameters were set to the same values as in the original run. Then the results of each run were matched to the grid stars to determine the completeness and any possible magnitude bias.

The test results for each field were used in choosing the cutoff magnitude $m_{c}$ at which the point source completeness was $\sim 90 \%$ and the photometric error small. Actually, the completeness depends on radius, so typically two different cutoff magnitudes were used, with $m_{c}$ being 0.5 mag fainter outside the central $\sim 1^{\prime}$. For each separate region of the image, an uncertainty in the completeness fraction $f_{c} \equiv \mathrm{N}_{\text {found }} / \mathrm{N}_{\text {add }}$ was calculated as $\delta f_{c}=\left[f_{c}\left(1-f_{c}\right) / \mathrm{N}_{\text {add }}\right]^{1 / 2}$ (Bolte 1989). In general, the corrections to point source counts are small $(<10 \%)$; more detail on the completeness tests is given by Blakeslee (1997b).

\subsubsection{Background Counts}

After rejecting extended objects and correcting for incompleteness, we fitted the radial distribution of point sources in each field to an $r^{1 / 4}$ law plus background model. The primary

reason for this procedure was to determine the background contamination from unresolved galaxies or faint stars. Many different binnings were explored for each field, and an average background value was chosen from among the fits. When the innermost points significantly changed the fit (due to a leveling off of the counts at small radii), they were excluded (similar to the power spectrum fits in $\$$ 3.5.1). A supplementary paper (Blakeslee et al. 1998) will discuss in more detail the point source photometry and radial distributions, including a comparison to the halo light distributions, along with similar data from a sample of southern BCGs. In the following section we discuss the power spectrum measurements and background estimates, and in $\$ 3.6$ we tabulate the point source densities and power spectrum normalizations, including background corrections.

\subsection{Fluctuation Reductions}




\subsubsection{Measuring the Power Spectra}

We use the same power spectrum analysis method as was used by BT95, and described in more detail by Tonry et al. (1990). After all objects brighter than the cutoff magnitude $m_{c}$ are masked out, the image power spectrum $P(k)$ is modeled as a linear function of the "expectation power spectrum" $E(k)$ :

$$
P(k)=P_{0} \times E(k)+P_{1} .
$$

$E(k)$ is computed as the convolution of the power spectra of the PSF and the window function of the mask. (The mask gets multiplied in image space, convolved in Fourier space.) $P_{0}$ is called the fluctuation power, the spatial variance in intensity which has been convolved with the PSF and therefore must have originated above the atmosphere. $P_{1}$ is the white noise component, which must be overcome by the signal. The star used for modeling the power spectrum of the PSF in each image was the same one used for doing the completeness tests.

The power spectra frequently show excess power at low wavenumber due to shells, tidal distortions, etc. which were not removed by the galaxy modeling, as well as from imperfect flattening at very low wavenumber. To deal with this problem, the low wavenumbers were omitted from the fits used to determine $P_{0}$. In order to determine the lowest usable (i.e., uncontaminated) wavenumber $k_{L}$, we fitted Eq. (9) to the data power spectrum over the interval $\left(k_{\min }, k_{\max }\right)$, setting $k_{\max }$ to the maximum wavenumber in the data and varying $k_{\min }$. The point at which the $P_{0}$ vs. $k_{\min }$ relation flattened out was adopted as $k_{L}$. Then, in an effort to eliminate some of the arbitrariness in the choice of $k_{L}$, we took a weighted average of the derived $P_{0}$ values from among the fits that had lowest wavenumbers ranging from $k_{\text {min }}=k_{L}$ to $k_{\min } \approx 2 k_{L}$; the rms variance in $P_{0}$ over these fits was used as an estimate of the uncertainty due to the choice of $k_{L}$. $P_{1}$ is so well determined by the high wavenumbers that it changes only negligibly among these fits.

Figure 2 provides an example of the power spectrum fits. The upper panel shows the power spectrum of an annulus of the A2197-2 image; the lower panel shows the fitted $P_{0}$ as a function of the lowest wavenumber used in the fit. (The power has been divided by the mean galaxy intensity to yield more manageable numbers.) The fits used in determining $P_{0}$ had lowest wavenumbers

ranging from 32 to 65 . The dashed line in the lower panel indicates the final value $P_{0}$ derived from an average of these fits.

\subsubsection{Globular Cluster and Background Fluctuations}

The mathematical formalism for this section was given by BT95; here, we describe the corrections for background variance applied in this sample. Two sources of contamination must taken into account: the stellar SBF and the fluctuations due to background galaxies. These must be subtracted from the $P_{0}$ measurements described in the previous section in order to determine the amplitude of the fluctuations due to GCs. 
Most of the observations reported here are in the $R$ band, unlike the Coma $I$-band observations of BT95, but the correction for the stellar SBF is done in the same way, relative to the measured value of the fluctuation magnitude in Virgo, where $\bar{m}_{R}=31.25$ for the BCG NGC 4472 and other big ellipticals (Tonry et al. 1990). We include a generous allowance of 0.30 mag for the uncertainty in $\bar{m}_{R}$ (intrinsic variations in $\bar{m}_{R}$ have not been well studied). The contribution from the uncertainty in the relative distances with respect to Virgo is included in the discussions of the calibrational errors in $\S .1$ and $\$ 4.2$.

While the mean $(R-I)$ color of the GCs is about 0.55 (Ajhar, Blakeslee, \& Tonry 1994), the galaxy color is $(R-I) \approx 0.68$, and the color of the SBF is $\left(\bar{m}_{R}-\bar{m}_{I}\right) \approx 1.6$ (Tonry et al. 1990). Thus, for a fixed value of $\left(m_{c}-m^{0}\right)$, the ratio of the power produced by faint GCs to the power from the stellar SBF improves by a factor of

$$
\begin{aligned}
\left(\frac{P_{G C}}{P_{S B F}}\right)_{R} \times\left(\frac{P_{G C}}{P_{S B F}}\right)_{I}^{-1} & =10^{-0.4\left[2(R-I)_{\mathrm{GC}}-\left(\bar{m}_{R}-\bar{m}_{I}\right)-(R-I)_{\mathrm{gal}}\right]} \\
& \approx 3.0 .
\end{aligned}
$$

(See Eqs. [10] and [12] of BT95.) The GC fluctuations in the $R$ band are roughly three times stronger relative to the SBF than they are in the $I$ band. Otherwise, this ratio of fluctuation powers scales with $m_{c}$ and distance modulus as shown in Figure 1 of BT95.

We included $K$-corrections in our estimates of the fluctuation magnitudes to account for the slightly different region of the rest frame spectrum that passes through the imaging filter as a function of galaxy redshift. The corrections were based on calculations done by Worthey (1996), who kindly redshifted his published stellar populations models (Worthey 1994). Unlike for the SBF Survey (Tonry et al. 1997), where $K_{I}(z)=7 \times z$ was used for the $\bar{m}_{I} K$-correction, the correction here was not well described by a linear function out to the redshift limit of this sample; however, a quadratic form proved adequate. The effect of the $K$-correction in the $R$ band is to make the estimate of the contamination from the stellar SBF smaller by $8 \%$ at $5000 \mathrm{~km} \mathrm{~s}^{-1}$ and by $13 \%$ at $10,000 \mathrm{~km} \mathrm{~s}^{-1}$, the limit of this survey. In practice, the stellar SBF contamination is only $\sim 10 \%$, so this small correction to it does not change the final results in any significant way, but it is important for avoiding bias. The uncertainty in the $K$-correction based on the scatter in Worthey's models was included in the calculation of our errors.

The larger correction $\left(\sim 10-50 \%\right.$, depending on $\left(m_{c}-m^{0}\right)$ and radius in the galaxy) to the measured variance is the one applied to account for the faint background galaxies. As in the SBF Survey and BT95, this is done by extrapolating the results from maximum likelihood routine (to be described by Ajhar et al. [1997]) which fits the magnitude distribution of the galaxies to a power law in the outer parts of the image, using the observed radial variation in the counts to subtract off the GCs. This background estimation can be a difficult and uncertain procedure when the galaxy nearly fills the image, but in such cases the relative size of the correction will be smaller. Out of necessity, the slope of the galaxy magnitude distribution is taken from Tyson (1988), and the normalization $T_{n}$ is fitted relative to his counts over the faintest few magnitudes of 
the image, in order to avoid possible dwarf satellites of the central galaxy. (Example outputs from the maximum likelihood program were shown by by Blakeslee [1997b]). From these measurements, the variance due to background galaxies is calculated according to Eq. (9) of BT95 and subtracted along with the $\mathrm{SBF}$ estimate from the measured variance $P_{0}$ in order to obtain $P_{G C}$, the variance due to GCs.

There is one other source of contamination to the variance measurement which one might consider, namely the possibility that a concentration of very faint dwarf galaxies may surround the BCG and contribute to $P_{0}$. For such dwarfs to cause problems, they would have to have a spatial distribution similar to the GCs (so as not to mimic the background galaxies) and a distribution in apparent magnitude extending fainter than $m_{c}$. For our sample, $\left(m_{c}-m^{0}\right)$ is typically $\sim 2.5$ mag; thus, the dwarfs would have to be fainter than about $M_{R}=-10.5$ (adopting a distance scale as in $\S 4.2$. The only known galaxies of such low luminosity are diffuse, low surface brightness dwarf speroidal (dSph) members of the Local Group (and possibly one faint member of Virgo, outside the cluster core [Durrell 1997]); these objects would surely be destroyed in the halo of a giant galaxy at the center of a rich cluster.

Coma is the only Abell cluster with a well studied faint-end of the galaxy luminosity function, and the data are basically similar to what is observed in Virgo (e.g., Ferguson \& Binggeli 1994). Thomas \& Gregory (1993) and Lobo et al. (1997) studied the dwarf galaxy luminosity function down to about $M_{R}=-16$ and $M_{R}=-14$, respectively. They both concluded that down to these luminosities, the dwarf elliptical (dE) and dSph populations followed the same spatial distribution as the larger cluster galaxies, except in the cluster core where the dSph galaxies had apparently been destroyed. (No significant population of gas-rich dwarf irregulars were detected.) Both sets of authors suggested that the faintest dwarfs in the cluster core may have been tidally destroyed or accreted. However, Bernstein et al. 1995 identified dwarf galaxies in the Coma core down to almost $M_{R}=-11$, although by this point the great majority of the objects were GCs, even $\sim 5^{\prime}$ $\left(\sim 100 h^{-1} \mathrm{kpc}\right)$ from the central galaxy.

Numerical simulations by Bassino et al. (1994) showed that the only surviving remants of dwarf satellite galaxies in the halo of an M87-like giant galaxy were the dense centers of nucleated $\mathrm{dE}$ galaxies. (Non-nucleated $\mathrm{dE}$ galaxies were destroyed entirely after a few orbits.) For nuclei with luminosities comparable to GCs to survive, they need to be about as dense as GCs, i.e., they would at that point essentially be GCs. Thus, we do not believe very faint dwarf satellite galaxies contaminate our variance measurement; the consistency of our results for the GCLFs presented in $\S 4.1$ support this conclusion. In the following section, we tabulate our measurements of $P_{0}$ and $P_{G C}$ along with the measured point source number densities. 


\subsection{Constraining $S_{N}$ and $\sigma$}

Table 5 summarizes the point source and fluctuation measurements, before and after background corrections. For each annular region of each galaxy, the table lists: bright cutoff magnitude $m_{b}$ of the point source counts; corrected number of point sources $N_{p s}\left(\operatorname{arcmin}^{-2}\right)$ fainter than $m_{b}$ but brighter than $m_{c}$; corrected number of GCs $N_{G C}\left(\operatorname{arcmin}^{-2}\right)$ over the same magnitude range following background subtraction; faint end cutoff magnitude $m_{c}$; fitted fluctuation power $P_{0}$ from objects fainter than $m_{c}$, in units of $10^{3}\left(e^{-} / \text {pixel }\right)^{2}$; background-subtracted power $P_{G C}$ due to GCs fainter than $m_{c}$, also in $10^{3}\left(e^{-} / \text {pixel }\right)^{2}$. The annular regions are defined as follows: c1, 32-64 pix; c2, 64-128 pix; c3, 128-256 pix; and c4, 256-512 pix.

In order to use these measurements to derive the total number of GCs around each program galaxy, and from that, the galaxy's specific frequency $S_{N}$, we must first estimate the value of the GCLF turnover $m^{0}$ in each galaxy. The value of $m_{V}^{0}$ in Virgo is $m_{V}^{0}$ (Virgo) $=23.75 \pm 0.05$ (Whitmore et al. 1995; Secker \& Harris 1993). Transforming to the $R$ band increases the uncertainty to $\sim 0.07$ mag. Although Virgo is too near to have been included in the Abell Catalog, its galaxy density is equivalent to that of a richness class 1 Abell cluster (Girardi et al. 1995), and it is therefore a valid calibrating object. The CMB velocity of Virgo is $v_{C M B}=1310 \pm 75 \mathrm{~km} \mathrm{~s}^{-1}$ (see $\S 3.2$ ), contributing an uncertainty of $0.12 \mathrm{mag}$ to the calibration. Combining the two sources of uncertainty in quadrature yields an estimated error in the $m^{0}$ calibration of $\sim 0.15$ mag. This systematic uncertainty will be left out of the error calculations for now, but we will discuss its effects later.

We adopt a value of $0.2 \mathrm{mag}$ as a best estimate of the intrinsic dispersion in $M^{0}$ among giant ellipticals in clusters (Harris 1996). It is also necessary to consider the possibility of Abell cluster radial peculiar velocities with respect to the CMB frame; these will have an effect on both $m^{0}$ and the estimated galaxy luminosity. There is a substantial literature on this subject (e.g. Aaronson et al. 1989; Huchra et al. 1990; Postman et al. 1992; Nichol et al. 1992; Zucca et al. 1993; Bahcall \& Oh 1996). We will adopt a compromise value of $400 \mathrm{~km} \mathrm{~s}^{-1}$ for the rms radial peculiar velocities of Abell clusters in the CMB frame. Thus, the random uncertainty in the estimated $m^{0}$ values for our sample galaxies is

$$
\begin{aligned}
\delta m^{0} & = \pm \sqrt{0.20^{2}+\left(\frac{5}{\ln 10} \times \frac{400}{c z_{C M B}}\right)^{2}} \\
& \approx \pm 0.26 \mathrm{mag}, \text { at } 5,000 \mathrm{~km} \mathrm{~s}^{-1} \\
& \approx \pm 0.22 \mathrm{mag}, \text { at } 10,000 \mathrm{~km} \mathrm{~s}^{-1}
\end{aligned}
$$

We follow the identical $\chi^{2}$ minimization procedure as BT95. For each of the four radial regions of each galaxy, we use the corrected variances and counts from Table 5 to calculate the $\chi^{2}$ 
values for a grid of points in the $N_{0}-\sigma$ plane:

$$
\chi^{2}=\left(\frac{N_{0}-N_{0}^{f l u}(\sigma)}{\delta N_{0}^{f l u}}\right)^{2}+\left(\frac{N_{0}-N_{0}^{c n t}(\sigma)}{\delta N_{0}^{c n t}}\right)^{2},
$$

where $N_{0}$ is the magnitude-integrated surface density of GCs and $\sigma$ is the Gaussian width of the GCLF; $N_{0}^{f l u}(\sigma)$ and $N_{0}^{c n t}(\sigma)$ are the values of $N_{0}$ determined from the fluctuations and counts, respectively, at a specific value of $\sigma$, and the denominators represent the uncertainties in these quantities. Thus, the grid points represent model GCLFs of the same $m^{0}$, determined for each cluster according to its CMB velocity and Eq. (8), but differing normalizations and widths. Blakeslee (1997b) showed the $\chi^{2}$ probability contours for each galaxy. Standard $K$-corrections (Schneider et al. 1983) were applied to the $m^{0}$ estimates before calculating $N_{0}^{f l u}$ and $N_{0}^{c n t}$, but these corrections are small, amounting to just $0.03 \mathrm{mag}$ in $R$ and $0.025 \mathrm{mag}$ in $I$ at the limit of the survey; the same corrections were made to the galaxy light, as it is very roughly the same color.

\section{RESULTS}

\subsection{GCLF Widths}

To determine final $\sigma$ values for each galaxy, we averaged the results from the $\chi^{2}$ minimizations for the useful regions of each galaxy, then varied $m^{0}$ according to Eq. (11), re-minimized $\chi^{2}$, and re-averaged. The uncertainty in $\sigma$ due to this (random) uncertainty in $m^{0}$ was added in quadrature to the internal error from the $\chi^{2}$ minimization. These two sources of uncertainty were usually comparable in size.

Figure 3 plots the derived $\sigma$ values from this analysis against their uncertainties. The two Coma galaxies from BT95 are included in the figure. (For values with asymmetric errorbars, the average of the errors is used.) The results cluster near $\sigma=1.4$ mag when the uncertainty is small, but drift higher when the uncertainty becomes large. Table 6 in the following section lists the individual values. The overall weighted mean is $\langle\sigma\rangle=1.45 \pm 0.03 \mathrm{mag}$, the median is $1.46 \mathrm{mag}$, and the unweighted mean is 1.49 mag with a dispersion of 0.13 mag. (Without the BT95 galaxies, the weighted mean, median, and unweighted values become 1.46, 1.49, and 1.50 mag, respectively, with the same dispersion.)

However, if we exclude the values of $\sigma$ with uncertainties greater than 0.15 mag, where the upward bias appears to set in, the weighted mean is $\langle\sigma\rangle=1.43 \pm 0.03 \mathrm{mag}$, and the median and unweighted mean are both $1.42 \mathrm{mag}$, with a $0.07 \mathrm{mag}$ dispersion. (Excluding the BT95 galaxies now makes no difference in these numbers.) It is not possible to eliminate this bias toward larger $\sigma$ by altering the GCLF $m^{0}$ calibration in any reasonable way. (For instance, adopting the Baum et al. [1997] calibration makes no difference in the size of the apparent bias.) We tentatively conclude that the GCLF width is the same for the whole sample. However, the more poorly determined values are biased towards larger $\sigma$, which causes one to wonder if there might also be 
some bias remaining in the mean of the low-error results. We consult the literature for insight into this question, then offer an explanation as to the most likely cause of the bias.

The primary GCLF calibrator for this analysis was M87, which is known to have $\sigma=1.40 \pm 0.06 \mathrm{mag}$ from HST measurements (Whitmore et al. 1995). Other well-measured GCLFs which provide reasonable comparisons for our sample are those of the Virgo BCG NGC 4472, $\sigma=1.47 \pm 0.10 \mathrm{mag}$ (Secker \& Harris 1993); the Fornax cD NGC 1399, $\sigma=1.38 \pm 0.09$ (Blakeslee \& Tonry 1996); the giant Virgo background elliptical NGC 4365, $\sigma=1.41 \pm 0.15$ (Forbes 1996, HST measurement); and NGC 5846, the central giant elliptical in a compact group, $\sigma=1.34 \pm 0.06$ (Forbes et al. 1996b, also with HST). Thus, we expect the galaxies in this sample to have $\sigma$ near $1.40 \mathrm{mag}$, as we found above for the well-constrained measurements. (Note that BT95 found $\sigma=1.70 \mathrm{mag}$ for M87, but that was with $m^{0}$ fixed to be $0.45 \mathrm{mag}$ too faint, based on the McLaughlin et al. [1994] results; setting $m^{0}$ to the proper value yielded a $\sigma$ of $1.45 \mathrm{mag}$ ).

In recent years, other functional forms besides Gaussians have been explored for fitting the GCLF. The most commonly used alternative is the " $t_{5}$ " distribution proposed by Secker (1992), but intersecting exponentials (Baum et al. 1995), tilted hyperbolic functions (Baum et al. 1997), and Gauss-Hermite expansions (Abraham \& van den Bergh 1995) have also been tried. What these alternate forms have in common is that their tails are broader than Gaussian, i.e., they are all "wingy", and they were all motivated by the smaller GCSs (200-300 members) of the Milky Way and M31. The GCLFs of large ellipticals with many thousand GCs, such as M87 (Whitmore et al. 1995) and NGC 1399 (Blakeslee \& Tonry 1996), are usually quite Gaussian. Thus, we would expect the GCLFs of our program galaxies to be Gaussian. As Abraham \& van den Bergh conclude, "for most applications, a simple Gaussian description is an adequate representation of the data."

However, the galaxies in our sample with poorly constrained and obviously biased measured values of $\sigma$ are the ones which are either among the smallest and relatively poorest in GCs (A634-1, A1016-1) or, in the case of A2162-1, suffered from poor seeing so that only the very brightest members far out in the tail contributed to the direct counts. If the counts are enhanced relative to the Gaussian model used for the $\chi^{2}$ minimization, the derived $\sigma$ will be too large. This was pointed out by BT95 and graphically illustrated in Figure 2-4 of Blakeslee (1997b). We believe this to be the most likely explanation for the bias in the under-constrained widths. A direct comparison in the following section of $S_{N}$ derived from counts and fluctuations with fixed $\sigma$ supports this view. The galaxies for which the counts penetrated to greater depths along the GCLF, or have no excess of bright GCs relative to a Gaussian, will not be similarly biased. The fact that the median $\sigma$ of the low-error measurements is $1.42 \mathrm{mag}$, virtually identical to the M87 calibrating GCLF value of $1.40 \mathrm{mag}$, supports this view. Moreover, it indicates that the working hypothesis of a universal GCLF for central galaxies in rich clusters has been vindicated.

To summarize, we have measured the GCLF widths of the Abell cluster galaxies in this sample. Although the under-constrained values are biased high, the remainder appear to be 
accurate. We find no evidence for any intrinsic dispersion among these better measured widths, and their average is very close to the value for M87, whose turnover magnitude $m^{0}$ and CMB velocity were used to estimate $m^{0}$ for the program galaxies, thereby allowing us to derive the GCLF widths. This result provides a valuable consistency check in support of the assumption of a universal GCLF for bright ellipticals in Abell clusters. In the previous section, we estimated the systematic uncertainty in the $m^{0}$ calibration to be \pm 0.15 mag, mainly due to the uncertainty in the CMB velocity of Virgo. Varying the estimates of $m^{0}$ by this amount has the effect of changing the individual GCLF widths by $0.04-0.06$ mag. Thus, we conclude that among the GCLF width measurements with internal uncertainties smaller than 0.15 mag, the mean is $\langle\sigma\rangle=1.43 \pm 0.03 \pm 0.05 \mathrm{mag}$, where the first errorbar reflects the internal error and the second indicates the systematic uncertainty. We note that if we had set the Virgo CMB velocity using the Baum et al. (1997) value of the relative Coma-Virgo distance, the derived values of $\sigma$ would be larger by $0.10-0.15 \mathrm{mag}$, and $\langle\sigma\rangle$ would increase by $0.12 \mathrm{mag}$. A discrepancy of this size would be difficult to resolve.

Because we find no evidence of significant differences in GCLF $\sigma$ between M87 and the sample galaxies or among the sample galaxies themselves, we report specific frequencies in the following section assuming the HST M87 value of $\sigma=1.40$ mag. For an intrinsic dispersion in this value, we assume $\pm 0.05 \mathrm{mag}$, consistent with our low-error results (which are in fact consistent with no intrinsic dispersion) and with the scatter in the measured widths of nearby dominant ellipticals. This approach was chosen because the derived $S_{N}$ is strongly anticorrelated with the value of $\sigma$. In this way, we hope to avoid biased intercomparisons among the $S_{N}$ results for these galaxies.

\subsection{Specific Frequencies}

If $N_{G C}$ is the total number of GCs in a galaxy and $M_{V}$ is its absolute $V$ magnitude, the specific frequency is $S_{N}=N_{G C} \times 10^{0.4\left(M_{V}+15\right)}$. In order to calculate $S_{N}$, one must adopt a zero point for the distance scale. Up to this point, all magnitudes have been set relative to their observed values in Virgo, using the Coma-Virgo relative distance to place Virgo in the CMB frame. We now adopt a Virgo distance modulus of 31.02 based on four Virgo spirals with Cepheid distance moduli (Freedman et al. 1996; there is also one about 1 mag more distant). Our assumptions imply a Hubble constant $H_{0} \sim 80 \mathrm{~km} \mathrm{~s}^{-1} \mathrm{Mpc}^{-1}$, but this is irrelevant for our primary goal of measuring trends in $S_{N}$. Transforming the $S_{N}$ values presented below to a

different distance scale requires multiplying them all by $10^{0.4\left[31.02-(m-M)_{0}\right]}$, where $(m-M)_{0}$ is the alternative Virgo distance modulus.

For each radial region of each galaxy, we assumed $\sigma=1.4$ mag and calculated the total GC surface density $N_{0}$ from the counts of GCs brighter than the cutoff magnitude $m_{c}$ and from the fluctuations resulting from those fainter than this cutoff. The two separate measurements were then weighted averaged, and all the GCs between some inner radial limit, discussed below, and an outer radius of $40 \mathrm{kpc}$ ( or $32 h^{-1} \mathrm{kpc}$ and $h=0.8$ ) were summed; the result was then divided 
by the normalized luminosity of the galaxy within the same radial range to derive the "metric $S_{N}$ " within $40 \mathrm{kpc}$. This was repeated for $\sigma=1.35$ and $1.45 \mathrm{mag}$, and then for $\sigma=1.4$ but with $m^{0}$ varied according to Eq. (11). (For $S_{N}$, unlike $\sigma$, the distance uncertainty due to random cluster velocities largely cancels, since it similarly effects both $N_{0}$ and the galaxy luminosity.) The variation in $S_{N}$ due to the uncertainties in these GCLF parameters was then added in quadrature to the internal errors which had been propagated from the counts and fluctuations. Table 6 lists the metric $S_{N}$ results, with their final errors, for each galaxy. (These were previously presented by Blakeslee [1997a,b].) It also lists the derived values of $\sigma$ discussed in the previous section, but these are not the ones used in calculating the tabulated values of $S_{N}$.

For reference, we list in Table 0 the specific frequency values $S_{N}^{f l u}$ and $S_{N}^{c n t}$ derived separately from the variance measurements and the counts, respectively, with GCLF $\sigma$ fixed at 1.40 mag. The internal measurement errors shown in the table include no allowance for uncertainty in the GCLF $m^{0}$ or $\sigma$, and are therefore uncorrelated. The value of the width $\sigma$ which would bring $S_{N}^{f l u}$ and $S_{N}^{c n t}$ into precise agreement for a given galaxy is the one listed in Table 6 for that galaxy. Table 0 also lists the number of standard deviations $\sigma_{d e v}$ separating the two $S_{N}$ values. Note that the four galaxies with $\sigma_{d e v}>2$ all have $S_{N}^{c n t}>S_{N}^{\text {flu }}$ and best-fit $\sigma \geq 1.53 \mathrm{mag}$ (Table 6), consistent with the bias in the GCLF width measurements being due to counts which are enhanced relative to a Gaussian model. However, we did not feel that this would justify throwing away the information provided by the counts.

Estimates of the global $S_{N}$ involve two very uncertain extrapolations of the GCS and galaxy profiles out to large radii. Not only are small uncertainties magnified by the extrapolations, but there is often no guarantee that the profiles do not change outside the imaged field. Thus, we chose not to make such extrapolations. The decision to report metric values of $S_{N}$ (i.e., values derived within the same physical radius around each galaxy) was made in order to avoid a bias in the reported $S_{N}$ with redshift. The metric radius of $40 \mathrm{kpc}$ was chosen because it corresponds to the limit of the image for the nearest of the sample galaxies (roughly 500 pix, or 2.'3, in the $1024^{2} R$-band images). Finally, the inner radius varied a bit and was set by practical constraints from the variable quality of the model subtraction at small radii. In effect, the inner limit was the smallest usable radius, typically smaller (in angle on the sky) for the more distant galaxies, but never less than $10^{\prime \prime}$, corresponding roughly to the expected GCS core radius in the nearest galaxies (Forbes et al. 1996a). It makes little difference, however, as such a small portion of the GCS is involved. Note that since $S_{N}$ is number per unit galaxy luminosity, if the GCs follow the same radial distribution as the halo light (true to at least first order), the "metric $S_{N}$ " will actually be independent of the chosen metric radius and will equal the global $S_{N}$. Henceforward, by $S_{N}$ we will mean these metric specific frequencies, unless otherwise stated.

The $S_{N}$ values listed in the table for A1656-1 (NGC 4889) and A1656-2 (NGC 4874) differ from the global values quoted in BT95 for two reasons. First, the RC2 photometry (de Vaucouleurs et al. 1976) used there was in error. It gave a total magnitude for NGC 4874 that was substantially too faint, as well as a slightly too faint total magnitude for NGC 4889. Using the photometry from 
the RC3 (de Vaucouleurs et al. 1991) yields global values of $S_{N}=10.2 \pm 2.4$ for NGC 4874 and $S_{N}=6.8 \pm 1.8$ for NGC 4889, closer to those shown in Table 6. Second, as stated above, the table gives the metric $S_{N}$ values within a limited radial range, using our own photometry of the galaxy light within that range. As the GC systems of those two galaxies were found to be more extended than the halo light, it is expected that their metric $S_{N}$ would be smaller than their global $S_{N}$, and we see from the table that this is the case.

We conclude with some comments on the measured values of $S_{N}$ for these galaxies and their uncertainties. Our results indicate the existence of a continuum of $S_{N}$ values, rather than a separation of galaxies into "normal" and "high" $S_{N}$ classes. For each of the three clusters with more than one galaxy in our sample, it is the second brightest galaxy that has the higher $S_{N}$ (although in the case of A539 the difference is not significant). These galaxies were included in the sample because they are all giant ellipticals with luminosities similar to those of the corresponding BCGs selected by LP. In fact, Table 1 shows that they all have larger $\alpha$ parameters than the BCGs, and comparison of their coordinates with those in Table 2 indicates that they are all closer to their cluster X-ray centers. Thus, for these three clusters, the most centrally dominant galaxy happens to be the second brightest one. We discuss below how central location in a cluster might affect $S_{N}$.

We have attempted to keep the $S_{N}$ errors listed in Table 6 both realistic and independent of each other. However, it is possible that they may include some systematic components, and therefore not be completely independent of each other. If, for example, the intrinsic (random) dispersion in $M^{0}$ is only $\sim 0.1$ mag instead of $\sim 0.2 \mathrm{mag}$, then the final errors will all be similarly overestimated, which is to say that they would contain a systematic component, and not be what we loosely term "independent". It seems unlikely, though, that the intrinsic dispersion in the GCLF width is less than the 0.05 mag we have adopted. The uncertainties from the GCLF parameters dominate, being 1-3 times as large as the uncertainties due to measurement error, depending on the size of the GC population and the depth of the data. The tabulated errors are offered as best estimates of the random (i.e., non-systematic) uncertainties in the individual $S_{N}$ values, but due to a preference to err on the side of caution, it is possible that these quoted random errors may be systematically overestimated.

Finally, we discuss the effects on the $S_{N}$ results of the estimated \pm 0.15 mag systematic uncertainty in the $m^{0}$ calibration. Increasing the individual $m^{0}$ estimates by 0.15 mag has the effect of increasing the derived $S_{N}$ values by $19 \pm 3 \%$; decreasing $m^{0}$ systematically by the same amount decreases the derived $S_{N}$ values by $15 \pm 2 \%$. Thus, the "top" of the $S_{N}$ continuum for this sample may move upward from 9.3 to 11.1, as the "bottom" moves from 3 to 3.6, or the top and bottom may decrease together to 7.9 and 2.5, respectively. These estimates assume that the galaxy luminosities are held fixed; varying them along with $m^{0}$ decreases the leeway to just a few percent, but the relative uncertainties in the Virgo distance modulus (which sets the zero point for the luminosities) and CMB velocity (which sets $m^{0}$ for each cluster with respect to Virgo) are similar in size. 


\subsection{Abell Cluster Inertial Frame Results}

The values of $S_{N}$ and GCLF width $\sigma$ reported and discussed in previous sections were all calculated in the CMB frame. However, there is another relevant frame which might also have been adopted, the Abell Cluster Inertial (ACI) frame. The ACI frame is defined to be at rest with respect to the large-scale Abell cluster bulk flow discovered by LP using the $L_{m}$ - $\alpha$ distance indicator.

In fact, we did the analysis twice simultaneously, using both CMB and ACI frame velocities to estimate $m^{0}$ and galaxy luminosities. The same distance zero point was used in both analyses; however, from one reference frame to the other, the relative distances between the galaxies change as a function of galactic coordinates, according to the transformation given by LP. Table 8 lists the $S_{N}$ and $\sigma$ results of our analysis in the ACI frame. The quantities were derived in an identical manner to those shown in Table 6, but the ACI frame velocities were used instead of CMB velocities. The $S_{N}$ values are generally higher. This is a consequence of the fact that the Coma-Virgo distance is fixed, but the velocity of Coma is nearly $10 \%$ smaller in the ACI frame (see Table 2).

Among the galaxies in clusters without fixed distances (i.e., everything but Coma), the fractional scatter in $S_{N}$ increases by $5 \%$ (and the absolute scatter increases by 11\%) when the ACI frame is used instead of the CMB frame. In addition, the mean and median GCLF $\sigma$ values among those with errors smaller than $0.15 \mathrm{mag}$ are now $\langle\sigma\rangle=1.48 \mathrm{mag}$ and $\sigma_{\text {med }}=1.51 \mathrm{mag}$, respectively; dropping the "fixed point" Coma galaxies, these values increase to 1.51 and 1.53 mag. (See Figure 4.) Thus, unlike the results for $\sigma$ in the CMB frame, which were discussed in $\S 4.1$, we do not find good agreement between the values of $\sigma$ in the ACI frame and the $\sigma=1.40$ mag width

of the GCLF $m^{0}$ calibrator M87. For these reasons, we have decided to concentrate the rest of the analysis on the results found using CMB frame velocities.

\section{5. $S_{N}$ CORRELATIONS}

The $S_{N}$ values derived in the CMB frame are plotted below against various properties of the host galaxy and surrounding cluster. To prevent confusion, we remind the reader that the word "cluster", when it appears alone, means "galaxy cluster"; the abbreviation "GC" refers to "globular cluster."

\subsection{Galaxy Properties}

The top panel of Figure 5 plots the derived $S_{N}$ values against absolute $R$-band CMB metric magnitude of the galaxy from LP. The lack of any significant correlation is understandable, as the LP metric magnitude is calculated within $12.5 \mathrm{kpc}$, while these $S_{N}$ values were derived for 
the total usable area of the galaxy within $40 \mathrm{kpc}$. Therefore, in the lower panel of the figure we plot $S_{N}$ against the absolute $V$ magnitude (since $S_{N}$ is defined relative to $\mathrm{V}$ magnitude) of the region of each galaxy in which the plotted $S_{N}$ was derived. Here, there does appear to be a weak correlation, but this is based almost entirely on the four faintest galaxies, which all have $S_{N} \lesssim 4$. Overall, the significance of the correlation is $\sim 0.95$, based on the Spearman rank-order correlation coefficient, but excluding the four faintest galaxies causes the significance level to drop to $\sim 0.40$ (i.e., no correlation). Galaxy luminosity is apparently not the driving force behind variations in $S_{N}$ among these galaxies. The panels of Figure 5 include the errorbars from Table 6, primarily to demonstrate their size, but the rest of the figures will omit them for the sake of clarity (and because of the possibility of systematically overestimated random errors, discussed above).

High values of $S_{N}$ have been associated with the phenomenon of extended cD halos (e.g., Harris 1991, McLaughlin et al. 1994). Figure 6 shows $S_{N}$ plotted against two measures of galaxy extent, the profile structure parameter $\alpha$ (defined in $\$ 2.1$ ), as listed by LP for the CMB frame, and the effective radius $R_{e}$ of the best fitting $r^{1 / 4}$-law, taken from Graham et al. (1996), who tabulated this quantity for the BCGs in the CMB frame with $H_{0}=80 \mathrm{~km} \mathrm{~s}^{-1} \mathrm{Mpc}^{-1}$. Where available, the effective radii for the non-BCGs were taken from the RC3 and brought into consistency with Graham et al. via the effective radius of the BCG, tabulated by both sources. Both panels exhibit a general upward trend. Since $\alpha$ measures the slope of the galaxy profile at fairly small radius, $R_{e}$ is probably a better measure of overall halo extent, but even with two apparent outliers, the top panel has less scatter. The biggest outlier in the $\alpha$ plot is A539-1 (with $\alpha \sim 0.5, S_{N}=9.1$ ); this is not the dominant galaxy in A539, judged by position relative to the cluster X-ray/dynamical center, although it does have many GCs. The other "outlier" is A2162-1 $\left(\alpha \sim 0.5, S_{N}=7.4\right)$, a $\mathrm{cD}$ whose extended halo only sets in at larger radius; it begins to move into line with the more extended galaxies in the lower panel. Although both plots show considerable scatter, they do support the view that $S_{N}$ and galaxy extent are somehow associated.

It is always advisable to look for systematic effects in final results; therefore in Figure 7 we have plotted our derived $S_{N}$ values against cluster redshift. These redshifts were the basis for our

$m^{0}$ estimates and the galaxy luminosity calculations, but as the figure shows, they do not correlate with $S_{N}$.

\subsection{Cluster Properties}

We now consider possible correlations of $S_{N}$ with properties of the clusters in which the galaxies reside. There are many different parameters to explore, including density, dynamics, morphology, and X-ray properties, any of which could have an effect on the GCS of the central galaxy in the cluster. Correlations of $S_{N}$ with some of the properties reflecting cluster mass or density have been discussed by Blakeslee (1997a), but here we provide a more complete discussion. We begin with the dynamics. 
In collecting cluster velocity dispersions from the literature, one must take care to ensure that the final set is a fairly homogenous one. Girardi et al. (1993) found that the various methods of estimating dispersions give consistent results as long as the number of cluster members with measured redshifts exceeds about 20; less sophisticated, or "robust," analyses often yield erroneous results with fewer than 15 measured redshifts. This collection of dispersions, listed in Table 2 , started with the "robust dispersions" determined by Girardi et al. (1993) from the data sets of Zabludoff et al. (1990) and Yahil \& Vidal (1977) for cluster galaxies within $1.5 h^{-1} \mathrm{Mpc}$ (the Abell radius, $r_{A}$ ) of the cluster centers. These dispersions have been superseded by the more recent measurements of Beers et al. (1991) for A569 and Zabludoff et al. (1993) for A1185, A1367, A1656, and A2199, both of whom likewise reported dispersions within $1 r_{A}$. To these have been added the dispersions for A2634 and A2666 from Scodeggio et al. (1995), who conducted a detailed study of these possibly interacting clusters. Because A2634 appears to be merging with a group of spirals at larger radius, and the smaller A2666 would otherwise be heavily contaminated by nearby A2634 galaxies, the dispersions within half an Abell radius have been used; these should more accurately reflect the central potentials of these clusters. All of these dispersions are based on at least 22 cluster members with measured velocities (after outliers have been rejected). Finally, Struble \& Rood (1991) list dispersions based on > 20 member redshifts for three other clusters in the BCG sample, so these have been used as well.

Figure $8 a$ shows the resulting plot of $S_{N}$ against cluster velocity dispersion. The tight correlation evident in the figure indicates that bright galaxies in regions of higher dispersion, and thus deeper potential wells, have more GCs per unit luminosity. In this and most of the following figures, the more central galaxies (judged by the X-ray center) in the clusters with multiple members in this sample are the ones shown as filled symbols, while the less central ones are shown as open symbols, since they often deviate from the correlations. The non-central galaxies will be referred to as "secondary", although three of the four are among the BCGs selected by LP, with the other one being NGC 4839, the third ranked galaxy in Coma. Correlations among cluster richness, dispersion, and X-ray temperature are well established (e.g., Bahcall 1981; Mushotzsky 1984; Edge \& Stewart 1991; Lubin \& Bahcall 1993; Girardi et al. 1993, 1996) and were to be expected as different measures of the cluster potential (assuming galaxies trace mass). Now we see a clear relationship between one of these quantities and something apparently unrelated, the GC specific frequency of the central bright galaxy in the cluster.

Recently, Girardi and collaborators have been studying cluster velocity dispersion profiles (Fadda et al. 1996; Girardi et al. 1996), reporting the asymptotic values at large radii. They argue that "asymptotic dispersions" are less affected by small-scale velocity anisotropies, and thus more indicative of the depth of the cluster potential as a whole. Because the profiles are usually peaked in the center before flattening further out, the asymptotic dispersions are generally lower than the central dispersions employed above. (These authors also cut the profiles of A2634 and A2666 off at smaller radii, however.) For this reason, we separately plot the asymptotic cluster velocity dispersions, as listed by Fadda et al., against $S_{N}$ in Figure $8 b$. The correlation is again very strong. 
Figure 9 shows that the correlation between $S_{N}$ and galaxy density as measured by Abell galaxy counts (Struble \& Rood 1987) is much weaker. The high formal significance of the correlation is due to the fact that the lowest $S_{N}$ central galaxies are all in relatively poor clusters; excluding these makes the correlation marginal. However, the uncertainties in the Abell counts are large and not well-known. The number density of galaxies drops steeply out to the $1 r_{A}$ limit, resulting in a large amount of background contaminaton, and making the counts a questionable measure of central density (Beers \& Tonry 1986). Bahcall (1981) showed that a better measure of the cluster density was provided by background-corrected counts of bright galaxies within $r_{A} / 2$. Those counts correlated better with cluster dispersion and X-ray luminosity than did the Abell counts. However, the present sample has very little overlap with the Bahcall sample; thus, we performed our own counts of bright galaxies within an even smaller radius, set by the size of the CCD field, making background correction completely unnecessary.

We counted all galaxies brighter than 0.05 and $0.1 L^{*}$ within several different radial distances from the program galaxy centers, where $L^{*}$ is the characteristic luminosity of the Schechter (1976) function. The value of $L^{*}$ was taken from Lin et al. (1996) and transformed from the $r$-band to our photometric bands following Schneider et al. (1983). The results of our counts are reported in Table 9 which lists the number of neighbors $N_{n}$ brighter than $0.1 L^{*}$ within 32 , 40, and $50 h^{-1} \mathrm{kpc}$ and the number brighter than $0.05 L^{*}$ within 32 and $40 h^{-1} \mathrm{kpc}$. Figure 10 plots the counts against $S_{N}$. We are hampered by small number statistics, but it is clear that the galaxies surrounded by more neighbors, or in regions of greater galaxy density, tend to have higher $S_{N}$. Again a relationship is suggested between central location within the cluster and $S_{N}$, assuming that the increase $N_{n}$ is the result of the galaxy's being closer to the cluster dynamical center.

Moving on to morphology, we plot $S_{N}$ against Bautz-Morgan type (from Abell et al. 1989) and Rood-Sastry type (Struble \& Rood 1987) in Figure 11. There have been reports based on smaller data sets that central galaxy $S_{N}$ correlates with BM type (McLaughlin, Harris, \& Hanes 1993, 1994; Harris, Pritchet, \& McClure 1995), in the sense of the "later" types (II-III, III) having higher $S_{N}$ central galaxies. The classification in this system is based on the relative dominance of the central galaxy in the cluster, with BM I clusters being dominated by single giant galaxies, and BM III cluster having no clearly dominant galaxy. McLaughlin et al. (1993) suggested that the apparent anti-correlation of $S_{N}$ with central galaxy dominance was due to dynamical evolution, with the central giants in the BM I and I-II clusters having diluted their originally high- $S_{N}$ down to lower levels through repeated mergers with other galaxies in the cluster. It was an interesting suggestion, but we do see not see a correlation of $S_{N}$ with BM type in this larger, more homogenous data set; thus, we find no evidence that such $S_{N}$ dilution is taking place.

Rood-Sastry type is also usually thought of in evolutionary terms, with the evolutionary state becoming more advanced along the following morphological sequence: Irregular, Flattened, Core-dominated, Linear, Binary, and cD. Again, our immediate reaction is that no correlation exists between central galaxy $S_{N}$ and RS type. However, Schombert \& West (1990) suggested that this classifications system reflected dynamical evolution only up to the $\mathrm{L}$ class, with the $\mathrm{B}$ and $\mathrm{cD}$ 
classes (clusters dominated by two giant galaxies and one giant galaxy, respectively) representing further evolution of the galaxy luminosity function following cluster virialization. This suggestion was based on an apparent correlation of the I-F-C-L sequence with supercluster environment. It is conceivable that there is a systematic change of $S_{N}$ along this subsequence of the RS system, but if so, it is most likely an ancillary consequence of the dependence central galaxy $S_{N}$ on cluster density, seen most clearly in the correlations with velocity dispersion (above) and X-ray properties (below).

From the correlation of $S_{N}$ with cluster velocity dispersion found in Figure 8, and the known relation between cluster velocity dispersion and the temperature of the X-ray emitting gas (e.g., Lubin \& Bahcall 1993) we expect some correlation between $S_{N}$ and cluster X-ray properties. Figures 12 shows $S_{N}$ plotted against cluster X-ray temperature $T_{X}(\mathrm{keV})$ and X-ray luminosity $L_{X}$ in the $0.5-4.5 \mathrm{keV}$ band (ergs sec${ }^{-1}$ ) from Jones \& Forman (1997). While previous investigations found no correlations of $S_{N}$ with these properties (Harris et al. 1995; West et al. 1995), our larger, more homogeneous data set clearly shows that there is a correlation, with a high level of significance, $\sim 0.998$.

The scatter in these plots is larger than in the velocity dispersion plots, but that is at least partially due to the larger uncertainties in the X-ray temperatures. If the observed variation of $S_{N}$ among these galaxies is driven by environmental density, then $S_{N}$ should correlate more strongly with $T_{X}$ than with $L_{X}$ because, assuming hydrostatic equilibrium, the gas temperature is determined simply by the depth of the cluster gravitational potential while the luminosity also depends on the amount of gas present. These two plots look so similar because half of the temperatures were estimated from the cluster $L_{X}-T_{X}$ relation (see Table 2), and there will be definite scatter about this relation. These estimated temperatures have $1 \sigma$ uncertainties of about $20 \%$, while the uncertainties in the velocity dispersions used above are $\lesssim 10 \%$.

West et al. (1995) have revived the idea of "intracluster globular clusters" (IGCs) which follow the overall cluster mass profile rather than being bound to individual galaxies. In this model, a giant galaxy which happens to lie near the cluster center will appear to have a high $S_{N}$ due to the "excess" intracluster GCs which become ipso facto associated with it. West et al. defined the excess to be the total number of GCs which elevate $S_{N}$ above a value of 4 , and assumed that this will be proportional to the projected matter density at the distance $r$ from the cluster X-ray center. For hydrostatic equilibrium and an isothermal potential, the excess will be proportional to $T_{X} /\left(1+r^{2} / r_{c}^{2}\right)$, where $r_{c}$ is the core radius of the cluster. In Figure 13, we plot the GC excess, defined relative to $S_{N}=3.5$, which more accurately reflects the lower $S_{N}$ limit for our sample, against $T_{X} /\left(1+r^{2} / r_{c}^{2}\right)$, where the $r_{c}$ values come from Jones \& Forman. Consistent with the IGC model, we find a roughly linear relationship. Even the non-central galaxies (open symbols) should follow this relationship, since it takes into account galaxy position in the cluster, and several of these galaxies do appear to deviate less than in the previous two plots, although otherwise there is no improvement. However, Blakeslee (1997a) showed that $S_{N}$ correlated marginally better with "local X-ray luminosity" $L_{X} /\left(1+r^{2} / r_{c}^{2}\right)$, than with $L_{X}$ itself. 
In the next section, we discuss the implications of the observed correlations, and noncorrelations, for the various theoretical models. We then describe how the correlations found here improve our understanding of GC systems, and the mechanisms which effect $S_{N}$ in Abell cluster central galaxies.

\section{DISCUSSION}

\subsection{Are High- $S_{N}$ BCGs "Special"?}

Among the bright cluster galaxies in this sample, $S_{N}$ varies continously as a function of certain well-defined galaxy and cluster parameters. Thus, the high- $S_{N}$ galaxies do not constitute a special class of object, but rather occupy the high end of the $S_{N}$ distribution. The total number of GCs appears to be determined primarily by cluster environmental factors, unlike the central galaxy luminosity, which is remarkably immune to variations in cluster properties (e.g., Hoessel, Gunn, \& Thuan 1980; PL).

As our results conflict with the common notion of "normal" and "anomalous" $S_{N}$ systems, we give some consideration to the galaxies in the literature which have inspired it. We begin with the prototypical high- $S_{N}$ galaxy M87. The most recent determination of $S_{N}$ for this galaxy was by McLaughlin et al. 1994, who reported a global value of $S_{N}=14.3 \pm 1.0$, before taking into account uncertainties in the distance or GCLF. Those authors adopted the same distance as we have; however, their fitted GCLF parameters significantly disagree with those determined with HST (Whitmore et al. 1995). This is understandable, as the fitted GCLF width and turnover are strongly correlated when the data do not go significantly fainter than $m^{0}$.

The much larger field of the McLaughlin et al. study makes their data preferable to HST for deriving $S_{N}$, and they provided a correction factor, a function of the GCLF parameters and distance modulus, that can be applied to their quoted $S_{N}$. Plugging the HST GCLF results into their correction formula, we find $S_{N}(M 87)=11 \pm 1$. Moreover, $S_{N}$ increases with radius in M87, so its "metric $S_{N}$ ", as we report for our sample, should be smaller than this global value. Thus, given the uncertainties, including the systematic error in the calibration of Virgo to the CMB frame, we can only conclude that M87 is at the high end of the continuous distribution in $S_{N}$ which we observe.

The other well-studied galaxy that has been numbered among the high- $S_{N}$ systems is the Fornax cD NGC 1399. This galaxy was first reported to have $S_{N} \sim 16$ (Bridges, Hanes, \& Harris 1991). A more recent study, using GCLF parameter which agree with ours (Blakeslee \& Tonry 1996) found $S_{N}=12 \pm 3$ (Kissler-Patig et al. 1996). However, this later study used a distance modulus that was 0.30 mag smaller than the new HST Cepheid result for this cluster (Silberman

et al. 1996). Using the Cepheid distance modulus, $S_{N}$ for this galaxy becomes $S_{N}(\mathrm{~N} 1399)=9 \pm 2$. The GCs and halo light follow similar distributions, so the metric $S_{N}$ would not differ much from 
the global value. This lies in the range of the galaxies studied here, though higher than we would expect for a cluster of low dispersion. On the other hand, Fornax is not a rich cluster (showing up as S373 in the supplementary catalog of southern poor clusters of Abell et al. [1989]), though it is very compact, so it is not clear how the $S_{N}$ of its central galaxy should compare to those studied here.

The rest of the high $S_{N}$ values found in the literature generally scale with the $S_{N}$ of M87. For instance, Harris et al. (1995) find $22 \pm 7$ and $13 \pm 6$ for the central cD galaxies in A2052 and A2107 (both outside the redshift limit of our survey; their other cluster, A2666, is discussed below). These numbers were derived relative to an assumed M87 value of 15; using the value of 11 implied by the HST GCLF yields $S_{N}(\mathrm{~A} 2052)=16 \pm 5$ and $S_{N}(\mathrm{~A} 2107)=9.5 \pm 4.4$, which are both still high, but not even the A2052 $S_{N}$ can be called anomalous, given the uncertainties.

Direct comparisons between our results and literature values are possible for several galaxies. BT95 used RC2 photometry and reported global values of $S_{N}$ which were in agreement with those found by Harris (1987), but we have revised those numbers down based on better photometry. In fact, Harris also used RC2 photometry, so his $S_{N}$ numbers get revised down in an identical manner; in particular, his value for NGC 4874 becomes $9 \pm 4.5$ when RC3 photometry is used. Butterworth \& Harris studied A1367-1 and reported the ratio $S_{N}(\mathrm{~A} 1367) / S_{N}(\mathrm{M} 87)$ as a function of radius. Reading the value of this ratio at our metric radius of $\sim 1$ ! 8 , and using the M87 $S_{N}$ from above gives $S_{N}=5.2 \pm 1$, very close to our value; even if we had used the larger $S_{N}$ of 14 for M87, we would agree with their results within the errors. For A2666-1, Harris et al. (1995) found $S_{N}=3 \pm 2$, again assuming the $S_{N}$ of 15 for M87. Using the smaller M87 value, their A2666-1 $S_{N}$ drops to $\sim 2.2$. In either case, it is close to our value of 3.5 for this galaxy. Finally, for the A2199 cD NGC 6166, Pritchet \& Harris (1990) reported $S_{N} \lesssim 4$, but a redetermination based on improved data found $S_{N}=9_{-4}^{+9}$ (Bridges et al. 1996). The latter result agrees with our value of $S_{N}=8.1$ for this galaxy.

In summary, for the few cases in which direct comparisons are possible, our results are in good agreement with published values. However, we believe that our larger, more homogenous data set affords a clearer view of the overall picture. We find that there is a continuum of possible $S_{N}$ values for bright cluster galaxies. Where a galaxy falls in this continuum may even be predictable from the cluster velocity dispersion, or $T_{X}$, and the projected distance of the galaxy from the cluster center.

\subsection{How Do the Models Compare?}

The observations presented here provide an unprecedented amount of information about $S_{N}$ variation among central galaxies in rich clusters; thus, we have the opportunity to evaluate the theoretical models from a more favorable vantage point than previously accessible. The history of GC formation theories is a long one, dating back at least to the work of Peebles \& Dicke (1968), 
but we will consider in turn only those extant models which purport to explain the wide variation of $S_{N}$ among central galaxies in clusters. A good model may even guide our understanding of the observations.

(1) Initial conditions. Until now, it was thought that no obvious correlations existed between the $S_{N}$ of the central cluster galaxy and other properties of either the galaxy or cluster (West et al. 1995). For instance, Harris et al. (1995), found no correlation of $S_{N}$ with cluster X-ray properties in a data set composed of their three galaxies and others from the literature, including central galaxies in 4 more Abell clusters, 3 AWM and MKW poor clusters, Fornax, and Virgo. They interpreted their results as indicating that GC formation in central cluster galaxies occurred early on, and reflected local initial conditions, thus the lack of any correlations. This view, that modern galaxies with high values of $S_{N}$ "were special ab initio" (van den Bergh 1984) and consequently formed their GCs "superefficiently" (McLaughlin et al. 1993), we call the "initial conditions" scenario. It goes back to Harris (1981) and is the prevailing view in the literature (e.g., Harris 1991).

In this scenario one must explain why some protogalaxies were privileged to undergo GC formation with enhanced efficiency. Harris \& Pudritz (1994) constructed a model for GC formation out of primordial pressure-confined, self-gravitating, magnetized supergiant molecular clouds. They hypothesized that larger external pressures may have prevailed in the proto-halos of $\mathrm{cD}$ galaxies and caused the primordial clouds in their model to fragment into more proto-GC cores per unit cloud mass. This idea remains speculative. In any case, our data indicate that many of the reasons for invoking initial conditions are no longer valid. In particular, it is not true that there are no correlations of $S_{N}$ with cluster properties. We find very good correlations between $S_{N}$ and cluster X-ray properties and with cluster velocity dispersion. Thus, we do not believe that the central galaxies in our study formed their GCs "superefficiently" as a result of local initial conditions.

(2) Biasing. A similarly motivated, but fundamentally different model is that of "biased GC formation." This is the apparent heir of the early work by Peebles \& Dicke, the major addition being that of dark matter. Examples of this include the models of Peebles (1984) and West (1993). In the biasing scenario, GC formation depends only on the height of the local density fluctuations with respect to some universal threshold. When the small scale (pc-sized) fluctuations are superposed on a larger scale (Mpc-sized), low amplitude fluctuation, such as might eventually evolve into a cluster of galaxies, the small scale fluctuations are more likely to broach this universal threshold; thus, they have a "bias" working in their favor. Biased formation may even produce GCs outside of any particular protogalaxy, with the resulting intergalactic GCs becoming associated with the whole of the cluster that forms there. Thus, the biasing model is also primordial, but less "local" than the initial conditions model. It predicts that $S_{N}$ should correlate strongly with the present-day density of the local environment, as we observe, but we are biased against this model because it is largely unconstrained. Biasing has been proposed as a possible mechanism at work in the IGC model discussed below; we will come back to it. 
(3) Mergers. In this model, gas-rich galaxies with low $S_{N}$ values, spirals in particular, merge to form elliptical galaxies, as suggested by Toomre (1977). In the process, GCs form out of shocked gas clouds, increasing the value of $S_{N}$ with every merger by an amount proportional to the available gas (Ashman \& Zepf 1992; Schweizer 1987). Repeated mergers of such systems in the core of a galaxy cluster might then yield high values of $S_{N}$. Kumai et al. (1993a,b) have discussed mechanisms for creating GCs in gas-rich collisions.

While mergers do indeed occur and massive star clusters may form in the process (e.g., Holtzman 1992), the question we must address is whether or not the observed variations in $S_{N}$ among central galaxies in clusters can be explained in this way. As formulated (Ashman \& Zepf 1992; Zepf \& Ashman 1993), the merger model predicts a strong dependence of $S_{N}$ on galaxy luminosity, which we do not see, while we do see other correlations which are not obvious in this picture. Thus, we do not believe that mergers of modern day galaxies can explain the observations. However, mergers of gaseous fragments in the protogalactic era, consistent with hierarchical structure formation models, almost certainly played a role in determining $S_{N}$, as we discuss in $\S 6.3$.

(4) Stripping. The idea that the central galaxy in the cluster potential increases its $S_{N}$ through preferential tidal stripping of the GCs from other galaxies in the cluster goes back to Forte, Martinez, \& Muzzio (1982). Muzzio (1987) reviewed the early work done on modeling such GCS dynamical evolution. Since the GC populations of elliptical galaxies tend to be more extended than the halo light (e.g. Harris 1991), a central galaxy might increase its $S_{N}$ through this process; BT95 noted that the number of GCs donated by other galaxies would not be unreasonable. The observed strong correlation of central galaxy $S_{N}$ with cluster velocity dispersion might occur as a result of stripping, since the crossing time in high dispersion clusters is lower and each galaxy would have passed through the core more times (although higher velocities also make stripping less efficient). Moreover, the correlations with local galaxy density and location of the galaxy relative to the cluster center are both easily understandable in the context of this model. One problem is that the increase of $S_{N}$ for the central cluster galaxy was too slow in the simulations reviewed by Muzzio (1987) to explain the observations. More modern simulations would be desirable. Stripping in the context of the IGC model is discussed below.

(5) Intracluster globular clusters (IGCs). The IGC model of West et al. (1995), mentioned above when we examined correlations of $S_{N}$ with cluster properties, simply proposes that there are large populations of GCs that belong to the cluster as a whole and follow the overall cluster density in their distribution. West et al. suggested stripping and biasing as possibilities for the origin of the IGCs.

As stated previously, our results appear consistent with the prediction of the IGC model that the number of GCs in excess of $S_{N} \sim 4$ should correlate with $T_{X} /\left(1+r^{2} / r_{c}^{2}\right)$. West et al. predicted that the $S_{N}$ values of two of our sample galaxies, A569-1 and A779-1, would be relatively low, $S_{N} \sim 4$, as we find in both cases. We believe that the IGC model is on the right track in treating 
the GC populations of the central galaxies as more a property of the cluster itself than of the galaxy. However, in order to clump around the galaxy, the IGCs must have a velocity dispersion closer to the internal dispersion of the galaxy $\left(\sim 300 \mathrm{~km} \mathrm{~s}^{-1}\right)$ than of the cluster $\left(\sim 750 \mathrm{~km} \mathrm{~s}^{-1}\right)$. This is observed to be the case for M87 where the dispersion of the GCs is $\sim 400 \mathrm{~km} \mathrm{~s}^{-1}$ (Cohen \& Ryzhov 1997). Moreover, the origin of the IGCs remains problematic. Biasing suffers the problems endemic to dark matter models in general, namely the lack of any useful external constraints. The alternative mechanism of stripping qualitatively explains our observations, but may not be efficient enough to produce large IGC populations. Therefore, it is not clear that the GCs are truly intergalactic; what we mean by saying that they are "more a property of the cluster" is that their number is determined by cluster properties, not by the properties of the central galaxy.

We conclude that no clear winner has emerged from among the available models, although there have been some strong contenders. In the following section we use as an additional guide the observationally known properties of the BCGs themselves. Along with the insight gained from our observations and this discussion, i.e., that the central galaxy GCs are more rightly considered a cluster property, we attempt to reach a coherent understanding of the observed variations in $S_{N}$.

\subsection{Another Model}

Discussions about why only certain central galaxies in clusters have "anomalously" high values of $S_{N}$ usually revolve around the presence of a $\mathrm{cD}$ envelope, which appears to be a helpful but not sufficient condition for having a high $S_{N}$. In an effort to uncover further clues in this regard, we extend the discussion to include another remarkable property of central cluster galaxies, namely their uniformity.

Sandage (1972) was the first to exploit the small dispersion in the metric luminosities $L_{m}$ of brightest cluster galaxies. He reported that $L_{m}$ defined within $\sim 20 h^{-1} \mathrm{kpc}$ had a dispersion of only $0.25 \mathrm{mag}$ for BCGs and was independent of cluster richness. Hoessel, Gunn, \& Thuan (1980) studied a larger, unbiased sample of BCGs and found an intrinsic dispersion of $\sim 0.35$ mag for $L_{m}$ defined within a $10 h^{-1} \mathrm{kpc}$. They also found that $L_{m}$ did become slightly brighter on average with cluster richness. Hoessel (1980) showed that this trend also correlated with the structure parameter $\alpha$, the slope of the luminosity profile at the metric radius. He defined the $L_{m^{-}} \alpha$ distance indicator, which had no residual dependence on richness. As Oegerle \& Hoessel (1991) showed, this indicator gives distances for BCGs that are nearly as good as fundamental plane distances but much less expensive observationally.

Most recently, Lauer \& Postman (LP 1994; PL 1995) have reinvestigated the use of the BCG $L_{m}-\alpha$ distance indicator for a sample of 119 BCGs, from which our own sample was selected. PL find that the intrinsic dispersion in $L_{m}$ drops from $0.33 \mathrm{mag}$ to 0.24 mag after the $\alpha$ correction

has been applied, and residuals about the $L_{m}-\alpha$ relation show no dependence on BCG luminosity, color, or location, nor on cluster richness. Moreover, they report that $L_{m}$ is independent of cluster 
richness even before the $\alpha$ correction has been applied. (Although Hudson \& Ebeling [1997] find evidence that both $L_{m}$ and residuals from the $L_{m}-\alpha$ indicator correlate with cluster X-ray luminosity.) They conclude that the small scatter in $L_{m}$ and $(B-R)$ color, coupled with the lack of any second-parameter effects, make BCGs "the most homogeneous distance indicators presently available for large-scale structure research."

In contrast to the uniformity of BCG metric luminosity, the $S_{N}$ values of BCGs have a range of more than a factor of three and are strongly correlated with cluster density as measured by velocity dispersion, X-ray temperature, and local galaxy density. Taken together, these points imply that high- $S_{N}$ BCGs are not anomalously rich in GCs, rather, they are underluminous with regard to their preeminent positions at the centers of rich clusters, while the numbers of GCs accurately reflect the dense environments.

To illustrate this view, Figure 14a plots absolute magnitudes within $40 \mathrm{kpc}$ (excluding galaxy center, as in the lower panel of Figure 5) against cluster velocity dispersion. There is a general trend, but the intrinsic scatter is large (the uncertainty in $M_{V}$ is $\sim 0.1 \mathrm{mag}$ ). In Figure 14 $b$, we show "GC excess" (the number of GCs which elevate $S_{N}$ above a value of 3.5) plotted against cluster dispersion. Despite the larger observational uncertainty, the scatter is much smaller, and the only discrepant points are non-central galaxies. Dividing the smoothly varying GC excesses in Figure 14b by the relatively invariant luminosities in Figure 14a (and adding 3.5) results in the observed correlations of $S_{N}$ with cluster density. The correlation of $S_{N}$ with $\alpha$ (Figure 6) can then be seen as a consequence of the fact that $\alpha$ itself correlates with cluster density (Blakeslee 1997a).

These observations might be explained by a model in which the GCs of these galaxies, like those of the Milky Way, formed early on and in proportion to the available mass. A later process, perhaps the collapse of the surrounding cluster, then halted the luminosity growth of the central galaxy. If this is true, then the number of GCs per unit total "background" (cluster) mass interior to a projected radius $R$ provides a more accurate description of the GC formation process than does the number per unit luminosity (i.e., $S_{N}$ ). We will attempt to estimate the cluster mass within $40 \mathrm{kpc}$, and so derive the number of GCs per unit mass, a quantity we call $\eta_{G C}$, for the galaxies in our sample.

The surface mass density near the center of a nonsingular isothermal sphere is $\Sigma_{c}=9 v_{r m s}^{2} / 2 G r_{c}$, where $v_{r m s}$ is the cluster velocity dispersion, and $r_{c}$ is the core radius. If $r_{c}$ were the same for all clusters, or were completely uncorrelated with $v_{r m s}$, then the mass enclosed within a fixed radius would go simply as the square of the velocity dispersion. However, there is a rough relationship between $v_{r m s}$ and $r_{c}$, in the sense that clusters with larger core radii tend to have higher velocity dispersions. Because of the difficulty in accurately measuring $r_{c}$ and the fact that a quarter of our clusters with known dispersions lack $r_{c}$ values, we will make use of this relationship. A straight fit for our sample gives $r_{c} \approx 2.5 v_{r m s}^{0.6}$, where $r_{c}$ is in kpc, $v_{r m s}$ is in $\mathrm{km} \mathrm{s}^{-1}$, and the scatter is about $50 \%$. Using this relation, we get the following expression for the 
total mass enclosed within $40 \mathrm{kpc}$ of the central galaxy

$$
M_{c}(R=40 \mathrm{kpc}) \approx 4.0 \times 10^{12}\left(\frac{v_{r m s}}{500 \mathrm{~km} \mathrm{~s}^{-1}}\right)^{1.4} M_{\odot} .
$$

Dividing the observed number of GCs within $40 \mathrm{kpc}$ by the mass implied by Eq. (13) yields the ratio $\eta_{G C} \equiv N_{G C} / M_{c}$, which we plot against cluster velocity dispersion in Figure 15 .

The figure demonstrates that $\eta_{G C}$ calculated according to this simple mass model is constant. The average value is about 0.7 per $10^{9} M_{\odot}$, with a scatter of $\sim 30 \%$. Thus, there appears to have been a single, universal formation efficiency for the GCs in our sample. For $10^{6} M_{\odot}$ GCs, the implied efficiency would be $\lesssim 0.1 \%$. Another way to describe the situation is to note that the observed $N_{G C}$ goes approximately as $v_{r m s}^{1.4}$. Because we wish to avoid models which postulate dark matter biasing or a density dependent efficiency, we hypothesize that the production of GCs per unit mass is constant. Finally, we have shown that a simple, plausible mass model produces the desired result.

\section{SUMMARY}

We have studied the largest sample to date of GC populations around central galaxies in Abell clusters, 7 times larger than any previous single study. The primary factor which allowed us to study such a large sample with a relatively small telescope was our use of the analysis methods developed by BT95. Our sample was selected from the BCG survey of Lauer \& Postman (1994); nearly all the new observations were made in the $R$-band, the one exception being that of A2199. For several clusters, the second brightest galaxy was included because it was comparable to the BCG in luminosity. In fact, in all of these cases, the second brightest galaxy was also more centrally located, as judged by both position relative to the X-ray center and the local galaxy density. In addition, a third Coma galaxy, NGC 4839 (A1656-3), was included by virtue of its cD envelope.

We detected a GC system and presented a measurement of the GCLF width $\sigma$ for each sample galaxy. A slight bias was found in the poorly constrained values of $\sigma$, but the more tightly constrained values showed little intrinsic dispersion and agreed closely with those found from deep imaging of nearby ellipticals, particularly M87, which was used as our " $m^{0}$ calibrator." We noted that our technique, as well as previous studies which used only direct counts, relied to some degree on the predictability of the GCLF turnover magnitude $m^{0}$. That is, the frequently made, but poorly tested, assumption of a universal GCLF is an important input. Thus, our GCLF results confirm the working assumption of a universal GCLF for central galaxies in the cores of rich clusters.

To limit biased comparisons of $S_{N}$ among sample galaxies, we assumed a single value of $\sigma$, consistent with our data, and calculated metric values of $S_{N}$, defined within $40 \mathrm{kpc}$. Our results for $S_{N}$ followed a continuous distribution; they were not segregated into "normal" and "high", 
or "anomalous", classes. This was the first time in which a continuum of $S_{N}$ values was clearly shown to exist. Contrary to the results of other studies, we find that $S_{N}$ correlates well with properties of the galaxy clusters, particularly central and asymptotic velocity dispersions, but also X-ray luminosity and temperature and the local galaxy density. For clusters with multiple galaxies in our sample, the one with the higher $S_{N}$ value was always the one which was more extended and closer to the cluster X-ray center. We did not find a strong correlation with cluster richness expressed in Abell counts, but that is not surprising, given their fairly qualitative and uncertain nature. We found no evidence of a previously proposed correlation of central galaxy $S_{N}$ with Bautz-Morgan class, and there is no obvious correlation with Rood-Sastry type either.

We discussed several models which have been proposed to explain the $S_{N}$ values of central cluster galaxies. Our data confirmed some predictions of the "intracluster globular cluster" (IGC) model, but the lack of a viable mechanism for producing the IGCs was judged problematic. The stripping model appeared most consistent with our data, but it may require an unacceptably long time scale for adding GCs to the central galaxy.

We concluded by offering our own explanation for the behavior of $S_{N}$ in these galaxies. We suggested that the GCs formed early, with their number $N_{G C}$ scaling in proportion to the available mass. (We do not exclude the possibility that their formation may have been episodic, yielding the frequently observed complex color/metallicity distributions.) The galaxy luminosity, on the other hand, is relatively independent of the cluster mass; perhaps the BCG formation was punctuated by the growth of the surrounding cluster in such a way as to produce the observed "standard candle" quality of these galaxies. In any case, the result is that $S_{N}$, the ratio of $N_{G C}$ to galaxy luminosity, is observed to scale with cluster mass in the same way as $N_{G C}$ itself does.

A better diagnostic of the physical processes affecting GC formation should therefore be provided by the observed number of GCs per unit "background" mass, a quantity which we have called $\eta_{G C}$. To estimate it, we adopted a simple flat core model and used it to calculate the total mass within the volume occupied by the GCs. We showed that the resulting $\eta_{G C}$ for our sample of galaxies was constant in this model. The implied "universal" formation efficiency is $\lesssim 1$ GC per $10^{9} M_{\odot}$. Thus, we believe that a consistent picture of the GC systems of central galaxies in clusters has begun to emerge, although further observational and theoretical work is needed in order to verify these results.

We thank Bob Barr for keeping MDM Observatory running smoothly and Paul Schechter for many helpful comments. We are grateful to Mark Postman and Tod Lauer for electronic data on their BCG sample, Guy Worthey for redshifted stellar populations calculations, Christine Jones-Forman for cluster X-ray information, and Jeff Willick for double-checking the Coma-Virgo relative distance from the Mark III Catalog. We thank Bjarne Thomsen, the referee, for numerous comments and suggestions that improved the paper. This research was supported by NSF grant AST94-01519, and the paper was completed under the sponsorship of a Caltech Fairchild Fellowship. 


\section{REFERENCES}

Aaronson, M. et al. 1989, ApJ, 338, 654

Abell, G. O. 1958, ApJS, 3, 211

Abell, G. O., Corwin H. G. Jr. \& Olowin, R. P. 1989, ApJS, 70, 1

Abraham, R. G. \& van den Bergh, S. 1995, ApJ, 438, 218

Ajhar, E. A., Blakeslee, J. P., \& Tonry, J. L. 1994, AJ, 108, 2087

Ajhar, E. A. et al. 1997, in preparation

Ashman, K. M. \& Zepf, S. E. 1992, ApJ, 384, 50

Bahcall, N. A. \& Oh, S. P. 1996, ApJ, 462, L49

Bahcall, N. A. 1981, ApJ, 247, 787

Bassino, L. P., Muzzio, J. C., \& Rabolli, M. 1994, ApJ, 431, 634

Baum, W. 1955, PASP, 67, 328

Baum, W. A. et al. 1995, AJ, 110, 2537

Baum, W. A., Hammergren, M., Thomsen, B., Groth, E. J., Faber, S. M., Grillmair, C. J., \& Ajhar, E. A. 1997, AJ, 113, in press

Beers, T. C., Forman, W., Huchra, J. P., Jones, C., \& Gebhardt, K. 1991, AJ, 102, 1581

Bernstein, G. M., Nichol, R. C., Tyson, J. A., Ulmer, M. P., \& Wittman, D. 1995, AJ, 110, 1507.

Beers, T. C. \& Tonry, J. L. 1986, ApJ, 300, 557

Blakeslee, J. P. 1997a, ApJ, 481, L59

Blakeslee, J. P. 1997b, PhD Thesis, MIT

Blakeslee, J. P. et al. 1998, in preparation

Blakeslee, J. P. \& Tonry, J. L. 1995, ApJ, 442, 579

Blakeslee, J. P. \& Tonry, J. L. 1996, ApJ, 465, L19

Bolte, M. 1989, ApJ, 341, 168

Bridges, T. J., Carter, D., Harris, W. E. \& Pritchet, C. J. 1996, MNRAS, 281, 1290

Bridges, T. J., Hanes, D. A., \& Harris, W. E. 1991, ApJ, 101, 469 
Burstein, D. \& Heiles, C. 1984, ApJS, 54, 33

Butterworth, S. T. \& Harris, W. E. 1992, AJ, 103, 1828

Cohen, J. G. 1988, AJ, 95, 682

Cohen, J. G. \& Ryzhov, Anton 1997, ApJ, in press

de Vaucouleurs, G., de Vaucouleurs, M., \& Corwin, H. G. 1976, Second Reference Catalog of Bright Galaxies (Austin: University of Texas Press), (RC2)

de Vaucouleurs, G., de Vaucouleurs, A., Corwin, H. G., Jr., Buta, R. J., Paturel, G., \& Fouqué, P. 1991, Third Reference Catalog of Bright Galaxies (New York: Springer), (RC3)

D’Onofrio, M., Capaccioli, M., Zaggia, S. R., \& Caon, N. 1997, MNRAS, in press astro$\mathrm{ph} / 9703157)$

Durrell, P. R. 1997, AJ, 113, 531

Edge, A. C. \& Stewart, G. C. 1991, MNRAS, 252, 428

Fadda, D., Girardi, M., Giuricin, G., Mardirossian, F., Mezzetti, M. \& Biviano, A. 1996, ApJ, 473,670

Ferguson, H. C. \& Binggeli B. 1994, A\&AR, 6, 67

Forbes, D. A. 1996, AJ, 112, 954

Forbes, D. A., Brodie, J., \& Huchra, J. P. 1996b, AJ, 112, 2448

Forbes, D. A., Franx, M., Illingworth, G. D., \& Carollo, C. M. 1996a, ApJ, 467, 126

Forte, J. C., Martinez, R. E., \& Muzzio, J. C. 1982, AJ, 87, 1465

Freedman, W. L., Madore, B. F., \& Kennicutt, R. C. 1996, in The Extragalactic Distance Scale, ed. M. Livio (Cambridge: Cambridge University Press), in press

Giovanelli, R. 1996, in The Extragalactic Distance Scale, ed. M. Livio (Cambridge: Cambridge University Press), in press

Girardi, M., Biviano, A., Giuricin, G., Mardirossian, F., \& Mezzetti, M. 1993, ApJ, 404, 38

Girardi, M., Biviano, A., Giuricin, G., Mardirossian, F., \& Mezzetti, M. 1995, ApJ, 438, 527

Girardi, M., Fadda, D., Giuricin, G., Mardirossian, F., Mezzetti, \& M. Biviano, A. 1996, ApJ, 457,61

Graham, A., Lauer, T. R., Colless, M., \& Postman, M. 1996, ApJ, 465, 534 
Harris, W. E. 1981, ApJ, 251, 497

Harris, W. E. 1987, ApJ, 315, L29

Harris, W. E. 1991, ARA\&A, 29, 543

Harris, W. E. 1996, in The Extragalactic Distance Scale, ed. M. Livio (Cambridge: Cambridge University Press), in press

Harris, W. E., Pritchet, C. J., \& McClure, R. D. 1995, ApJ, 441, 120

Harris, W. E. \& Pudritz, R. E. 1994, ApJ, 429, 177

Harris, W. E. \& van den Bergh, S. 1981, AJ, 86, 1627

Hoessel, J. G. 1980, ApJ, 241, 493

Hoessel, J. G., Gunn, J. E., \& Thuan T. X. 1980, ApJ, 241, 486

Holtzman, J. et al. 1992, AJ, 103, 691

Hubble, E. 1932, ApJ, 76, 44

Huchra, J. P., Henry, J. P., Postman, M., \& Geller, M. J. 1990, ApJ, 365, 66

Hudson, M. J. \& Ebeling, H. 1997, ApJ, 479, 621

Jones, C. \& Forman, W. 1997, in preparation

Kissler-Patig, M., Kohle, S., Hilker, M., Richtler, T., Infante, L., Quintana, H. 1996, A\&A, in press

Kumai, Y., Basu, B., \& Fujimoto, M. 1993a, ApJ, 404, 144

Kumai, Y., Hashi, Y., \& Fujimoto, M. 1993b, ApJ, 416, 576

Landolt, A. U. 1992, AJ, 104, 340

Lauer, T. R. \& Postman, M. 1994, ApJ, 425, 418

Lauer, T. R. 1988, ApJ, 325,49

Lin, H., Kirshner, R. P., Shectman, S. A., Landy, S. D., Oemler, A., Tucker, D. L., \& Schechter, P. L., 1996, ApJ, 464, 60

Lobo, C., Biviano, A. Durret, F., Gerbal, D., Le Fèvre, O., Mazure, A., \& Slezak, E. 1997, A\&A, 317,385

Lubin, L. M. \& Bahcall, N. A. 1993, ApJ, 415, L17 
McLaughlin, D. E., Harris, W. E., \& Hanes, D. A. 1993, 409, L45

McLaughlin, D. E., Harris, W. E., \& Hanes, D. A. 1994, 422, 486

Mushotzsky, R. 1984, Phys. Scripta, T7, 157

Muzzio, J. C. 1987, PASP, 99, 245

Nichol, R. C., Collins, C. A., Guzzo, L., \& Lumsden, S. L. 1992, MNRAS, 255, 24p

Nilson, P. 1973, Uppsala General Catalogue of Galaxies (Uppsala: Reg. Soc. Scient. Ups.)

Oegerle, W. R. \& Hoessel, J. G. 1991, ApJ, 375, 15

Peebles, P. J. E. 1984, ApJ, 277, 470

Peebles, P. J. E. \& Dicke, R. H. 1968, ApJ, 154, 891

Postman, M. 1996, private communication

Postman, M., Huchra, J. P. \& Geller, M. J. 1992, ApJ, 384, 404

Postman, M. \& Lauer, T. R. 1995, ApJ, 440, 28

Pritchet, C. J. \& Harris, W. E. 1990, ApJ, 355, 410

Pritchet, C. J. \& van den Bergh, S. 1985, AJ, 90, 2027

Racine, R. 1968a, PASP, 80, 326

Racine, R. 1968b, JRASC, 62, 367

Sandage, A. 1972, ApJ, 178, 1

Schechter, P. L. 1976, ApJ, 203, 297

Schechter, P. L., Mateo, M., \& Saha A. 1993, PASP, 105, 1342

Schneider, D. P., Gunn, J. E., \& Hoessel, J. G. 1983, ApJ, 264, 337

Schweizer, F. 1987, in Nearly Normal Galaxies, ed. S. M. Faber (New York: Springer), 18

Schombert, J. M. 1988, ApJ, 328, 475

Schombert, J. M. \& West, M. J. 1990, ApJ, 363, 331

Scodeggio, M., Solanes, J. M., Giovanelli, R., \& Haynes, M. P. 1995, ApJ, 444, 41

Secker, J. 1992, AJ, 104, 1472

Secker, J. \& Harris, W. E. 1993, AJ, 105, 1358 
Shopbell, P. L., Bland-Hawthorn, J., \& Malin, D. F. 1993, AJ, 106, 1344

Silbermann, N.A. et al. 1996, in The Extragalactic Distance Scale, ed. M. Livio (Cambridge: Cambridge University Press), in press

Smith, M. G. \& Weedman, D. W. 1976, ApJ, 205, 709

Struble, M. F. \& Rood, H. J. 1987, ApJS, 63, 555

Struble, M. F. \& Rood, H. J. 1991, ApJS, 77, 363

Thompson, L. A. \& Gregory, S. A. 1993, AJ, 106, 2197

Thompson, L. A. \& Valdes, F. 1987, ApJ, 315, L35

Tonry, J. L. 1987, in Structure and Dynamics of Elliptical Galaxies, ed. T. de Zeeuw (Dordrecht: D. Reidel)

Tonry, J. L., Ajhar, E. A., \& Luppino, G. A. 1990, AJ, 100, 1416

Tonry, J. L., Blakeslee, J. P., Ajhar, E. A., \& Dressler, A. 1997, ApJ, 475, 399

Tonry, J. L. \& Schneider, D. P. 1988, AJ, 96, 807

Toomre, A., 1977, in The Evolution of Galaxies and Stellar Populations, eds. B. M. Tinsley and R. B. Larson (New Haven: Yale Univ. Observatory), 401

Tyson, J. A. 1988, AJ, 96, 1

van den Bergh, S. 1984, PASP, 96, 329

van den Bergh, S. 1992, PASP, 104, 861

van den Bergh, S., Pritchet, C. J., \& Grillmair, C. G. 1985, AJ, 90, 595

West, M. J. 1993, MNRAS, 265, 755

West, M. J., Côté, P., Jones, C., Forman, W., \& Marzke, R. O. 1995, ApJ, 453, L77

Whitmore, B. C. 1996, in The Extragalactic Distance Scale, ed. M. Livio (Cambridge: Cambridge University Press), in press

Whitmore, B. C., Sparks, W. B., Lucas, R. A., Macchetto, F. D., \& Biretta, J. A. 1995, ApJ, 454, L73

Willick, J. A. 1997, private communication

Willick, J. A., Courteau, S., Faber, S. M., Burstein, D., Dekel, A., \& Strauss, M. A. 1997, ApJS, 109,333 
Worthey, G. 1994, ApJS, 95, 107.

Worthey, G. 1996, private communication

Yahil, A. \& Vidal, N. 1977, ApJ, 214, 347

Zabludoff, A. I., Geller, M. J., Huchra, J. P., \& Ramella, M. 1993, AJ, 106, 1273

Zabludoff, A. I., Huchra, J. P., \& Geller, M. J. 1990, ApJS, 74, 1

Zepf, S. E. \& Ashman, K. M. 1993, MNRAS, 264, 611

Zucca, E., Zomorani, G. Scaramella, R., \& Vettolani, G. 1993, ApJ, 407, 470

Zwicky, F. 1968, Catalogue of Galaxies and of Clusters of Galaxies (Pasadena: California Institute of Technology) 
Table 1. The BCG Sample

\begin{tabular}{|c|c|c|c|c|c|c|c|}
\hline Abell & RA (J2000) Dec & $b$ & $c z_{h}$ & $A_{B}$ & $M_{R, m}$ & $\alpha$ & Name \\
\hline $262-1$ & $015246+360905$ & $136.57-25.09$ & 4831 & .24 & -22.189 & .810 & N0708 \\
\hline $347-1$ & $022526+414927$ & $141.11-17.71$ & 5257 & .24 & -22.352 & .601 & N0910 \\
\hline $397-1$ & $025629+155459$ & $161.81-37.34$ & 10286 & .27 & -22.542 & .582 & C463-037 \\
\hline $539-1$ & $051655+063310$ & $195.65-17.60$ & 9682 & .51 & -22.484 & .511 & C421-019 \\
\hline $539-2$ & $051637+062628$ & $195.71-17.72$ & 8318 & .51 & -22.210 & .785 & U03274 \\
\hline $569-1$ & $070908+483655$ & $168.58+22.80$ & 5724 & .34 & -22.418 & .486 & N2329 \\
\hline $634-1$ & $081545+581916$ & $159.06+33.79$ & 8135 & .13 & -22.258 & .498 & U04289 \\
\hline $779-1$ & $091947+334459$ & $191.09+44.39$ & 6867 & .00 & -22.858 & .594 & $\mathrm{~N} 2832^{\dagger}$ \\
\hline $999-1$ & $102324+125006$ & $227.94+52.58$ & 9749 & .11 & -22.267 & .441 & C065-015 \\
\hline 1016-1 & $102708+110037$ & $231.29+52.48$ & 9705 & .03 & -22.048 & .430 & I0613 \\
\hline $1177-1$ & $110944+214532$ & $220.44+66.29$ & 9561 & .00 & -22.453 & .724 & U06203 \\
\hline $1185-1$ & $111038+284603$ & $202.81+67.72$ & 10521 & .00 & -22.444 & .616 & N3550 \\
\hline $1314-1$ & $113449+490438$ & $151.77+63.54$ & 9977 & .00 & -22.461 & .583 & I0712 \\
\hline $1367-1$ & $114402+195659$ & $234.29+72.99$ & 6237 & .00 & -22.496 & .518 & N3842 \\
\hline $1656-1$ & $130008+275836$ & $57.19+87.89$ & 6497 & .05 & -22.957 & .590 & N4889 \\
\hline $1656-2$ & $125936+275733$ & $58.08+88.01$ & 7176 & .05 & -22.545 & .855 & $\mathrm{~N} 4874^{\dagger}$ \\
\hline $1656-3$ & $125725+272948$ & $48.79+88.62$ & 7335 & .03 & -22.287 & .610 & $\mathrm{~N} 4839^{\dagger}$ \\
\hline $2162-1$ & $161236+292904$ & $48.33+46.01$ & 9547 & .07 & -22.475 & .503 & N6086 ${ }^{\dagger}$ \\
\hline $2197-1$ & $162945+404842$ & $64.68+43.51$ & 8800 & .00 & -22.887 & .586 & N6173 \\
\hline $2197-2$ & $162741+405537$ & $64.84+43.90$ & 9408 & .01 & -22.350 & .702 & N6160* \\
\hline 2199-1 & $162838+393303$ & $62.93+43.69$ & 9348 & .00 & -22.657 & .755 & N6166 ${ }^{\dagger}$ \\
\hline 2634-1 & $233829+270150$ & $103.50-33.07$ & 9141 & .16 & -22.748 & .650 & $\mathrm{~N} 7720^{\dagger}$ \\
\hline $2666-1$ & $235059+270848$ & $106.72-33.81$ & 8123 & .13 & -22.768 & .549 & N7768 \\
\hline
\end{tabular}

${ }^{\dagger}$ Schombert (1988) cD galaxy.

*Selected as BCG by Hoessel, Gunn, \& Thuan (1980). 
Table 2. Abell Cluster Information

\begin{tabular}{|c|c|c|c|c|c|c|c|c|c|}
\hline Abell & $\mathrm{RA}_{X}(\mathrm{~J} 2000) \operatorname{Dec}_{X}$ & $c z_{h}$ & $c z_{C}$ & $c z_{A}$ & $\sigma$ & $T_{X}$ & $\mathrm{R}$ & $\mathrm{BM}$ & $\mathrm{RS}$ \\
\hline 262 & $15246+360836$ & 4913 & 4659 & 5310 & 498 & 2.4 & 0 & III & $\mathrm{C}$ \\
\hline 347 & 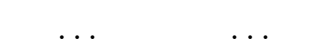 & 5604 & 5391 & 6000 & 582 & & 0 & II-III & $\mathrm{C}$ \\
\hline 397 & $25638+155338$ & 9975 & 9765 & 10560 & $\ldots$ & $1.6^{*}$ & 0 & III & $\mathrm{F}$ \\
\hline 539 & $51636+062630$ & 8754 & 8755 & 9390 & 787 & 3.0 & 1 & III & $\mathrm{F}$ \\
\hline 569 & $70911+483658$ & 5749 & 5832 & 6060 & 374 & $1.4^{*}$ & 0 & II & B \\
\hline 634 & $81434+580252$ & 8135 & 8234 & 8280 & 309 & $0.9^{\dagger}$ & 0 & III & $\mathrm{F}$ \\
\hline 779 & $91947+334449$ & 6796 & 7039 & 6930 & 472 & $1.5^{*}$ & 0 & I-II & $\mathrm{cD}$ \\
\hline 999 & $102323+125013$ & 9603 & 9942 & 9600 & 417 & $1.2^{*}$ & 0 & II-III & $\mathrm{L}$ \\
\hline 1016 & $102703+105842$ & 9669 & 10013 & 9660 & 247 & $1.3^{\dagger}$ & 0 & 列 & $\mathrm{L}$ \\
\hline 1177 & . & 9561 & 9885 & 9420 & $\ldots$ & $\ldots$ & 0 & I & $\mathrm{cD}$ \\
\hline 1185 & $111045+284246$ & 9917 & 10217 & 9780 & 718 & 3.9 & 1 & II & $\mathrm{C}$ \\
\hline 1314 & $113448+490510$ & 9838 & 10043 & 9690 & $\ldots$ & 5.0 & 0 & III & $\mathrm{C}$ \\
\hline 1367 & $114440+194235$ & 6469 & 6795 & 6240 & 802 & 3.5 & 2 & II-III & $\mathrm{F}$ \\
\hline 1656 & $125943+275612$ & 6961 & 7229 & 6570 & 1140 & 8.1 & 2 & II & $\mathrm{B}$ \\
\hline 2162 & $\ldots$ & 9629 & 9689 & 9030 & $\ldots$ & $\ldots$ & 0 & II-III & I \\
\hline 2197 & $162740+405539$ & 9042 & 9065 & 8520 & 589 & $1.6^{*}$ & 1 & III & $\mathrm{L}$ \\
\hline 2199 & $162838+393310$ & 9034 & 9059 & 8490 & 823 & 4.7 & 2 & I & $\mathrm{cD}$ \\
\hline 2634 & $233825+270056$ & 9153 & 8807 & 9330 & 800 & 3.4 & 1 & II & $\mathrm{cD}$ \\
\hline 2666 & $235101+270825$ & 8057 & 7714 & 8250 & 380 & $0.9^{*}$ & 0 & I & $\mathrm{cD}$ \\
\hline
\end{tabular}

*Temperature estimated from X-ray luminosity.

${ }^{\dagger}$ Upper limit.

Table 3. Observing Runs

\begin{tabular}{cccccccl}
\hline \hline Run & Detector & " $/$ pix & $\mathrm{F}$ & $m_{1}$ & $A$ & $C$ & \multicolumn{1}{c}{ Notes } \\
\hline 0593 & Loral $2048^{2}$ & 0.343 & $I$ & 24.349 & 0.140 & 0.030 & Coma; $2 \times 2$ \\
0794 & ${\text { STIS } 2048^{2}}^{2}$ & 0.240 & $I$ & 24.034 & 0.085 & 0.020 & N6166 \\
1194 & Tek 1024 & 0.275 & $R$ & 25.582 & 0.098 & 0.011 & poor weather \\
0295 & Tek 1024 & 0.275 & $R$ & 25.565 & 0.111 & 0.015 & \\
0395 & Tek 1024 & 0.275 & $R$ & 25.530 & 0.115 & 0.014 & bright moon \\
0995 & Tek 1024 & 0.275 & $R$ & 25.340 & 0.117 & 0.015 & \\
\hline
\end{tabular}


Table 4. Galaxy Observations

\begin{tabular}{rrrrrrrr}
\hline \hline Galaxy & Run & Exp & PSF & $m_{1}^{*}$ & $\mu_{\text {sky }}$ & SNR $^{0}$ & $\mathrm{~N}_{\text {obj }}$ \\
\hline A262-1 & 1194 & 5600 & 1.10 & 34.681 & 20.75 & 4.4 & 1394 \\
A347-1 & 1194 & 4000 & 0.99 & 34.348 & 20.67 & 3.0 & 1320 \\
A347-1 & 0995 & 4200 & 0.86 & 34.145 & 21.05 & 3.8 & 2351 \\
A397-1 & 0995 & 20700 & 0.87 & 35.852 & 20.29 & 1.8 & 1350 \\
A539-1 & 0295 & 11900 & 0.93 & 35.348 & 20.92 & 2.2 & 1300 \\
A539-2 & 0295 & 10850 & 0.89 & 35.250 & 20.77 & 2.0 & 1511 \\
A569-1 & 0295 & 4200 & 0.96 & 34.335 & 21.18 & 3.3 & 1210 \\
A569-1 & 0395 & 6000 & 1.24 & 34.656 & 20.96 & 2.7 & 867 \\
A634-1 & 0295 & 12600 & 0.98 & 35.626 & 21.24 & 3.1 & 1796 \\
A779-1 & 0295 & 6600 & 0.90 & 35.018 & 21.14 & 3.3 & 2086 \\
A999-1 & 0295 & 13200 & 0.88 & 35.679 & 21.12 & 2.3 & 1802 \\
A1016-1 & 0295 & 11700 & 0.86 & 35.597 & 21.09 & 2.2 & 1590 \\
A1177-1 & 0295 & 15025 & 0.96 & 35.903 & 21.14 & 2.4 & 2157 \\
A1185-1 & 0395 & 23100 & 0.91 & 36.310 & 19.78 & 1.5 & 1423 \\
A1314-1 & 0395 & 19500 & 1.03 & 36.120 & 21.21 & 2.4 & 2222 \\
A1367-1 & 0295 & 4200 & 0.77 & 34.501 & 20.77 & 2.7 & 2113 \\
A1656-1 & 0593 & 4900 & 0.88 & 33.392 & 19.02 & 0.9 & 1216 \\
A1656-2 & 0593 & 5500 & 0.83 & 33.542 & 19.10 & 1.1 & 1663 \\
A1656-3 & 0395 & 10800 & 1.00 & 35.479 & 19.95 & 2.0 & 1429 \\
A2162-1 & 0995 & 18900 & 1.29 & 35.789 & 20.59 & 1.3 & 1196 \\
A2197-1 & 0395 & 19300 & 0.99 & 36.127 & 20.03 & 1.8 & 1210 \\
A2197-2 & 0395 & 12800 & 1.05 & 35.689 & 21.26 & 2.5 & 1786 \\
A2199-1 & 0794 & 11700 & 0.92 & 34.135 & 19.82 & 1.1 & 2530 \\
A2634-1 & 0995 & 13700 & 1.01 & 35.428 & 20.04 & 1.4 & 1455 \\
A2666-1 & 1194 & 8200 & 0.91 & 35.023 & 20.83 & 2.4 & 1036 \\
\hline
\end{tabular}


Table 5. Point Source Counts and Variance Measurements

\begin{tabular}{|c|c|c|c|c|c|c|c|c|c|c|}
\hline Gala & $m_{b}$ & $N_{p s}$ & \pm & $N_{G C}$ & \pm & $m_{c}$ & $P_{0}$ & \pm & $P_{G C}$ & \pm \\
\hline \multicolumn{11}{|c|}{ Runs $0794 \& 1194}$. \\
\hline A262-1.c1 & 21.0 & 97.6 & 28.1 & 76.6 & 28.3 & 24.0 & 1090 & 53 & 931 & 75 \\
\hline A262-1.c2 & 21.0 & 76.3 & 10.1 & 55.3 & 10.8 & 24.0 & 546 & 24 & 415 & 57 \\
\hline A262-1.c3 & 21.0 & 50.9 & 4.6 & 29.9 & 5.9 & 24.0 & 371 & 15 & 253 & 53 \\
\hline A262-1.c4 & 21.0 & 27.2 & 1.8 & 6.2 & 4.1 & 24.0 & 201 & 18 & 88 & 54 \\
\hline A347-1.c1 & 21.0 & 122.8 & 26.3 & 102.0 & 26.4 & 24.0 & 688 & 39 & 606 & 43 \\
\hline A347-1.c2 & 21.0 & 54.9 & 8.4 & 34.1 & 8.6 & 24.0 & 313 & 17 & 243 & 24 \\
\hline A347-1.c3 & 21.0 & 30.5 & 3.2 & 9.7 & 3.7 & 24.0 & 189 & 4 & 123 & 17 \\
\hline A347-1.c4 & 21.0 & 22.5 & 1.4 & 1.7 & 2.3 & 24.0 & 103 & 7 & 39 & 18 \\
\hline A2199-1.c2 & 21.5 & 100.0 & 15.6 & 74.0 & 15.7 & 24.0 & 311 & 29 & 262 & 31 \\
\hline A2199-1.c3 & 21.5 & 68.9 & 6.0 & 42.9 & 6.2 & 24.0 & 151 & P & 107 & 12 \\
\hline A2199-1.c4 & 21.5 & 36.1 & 2.1 & 10.1 & 2.6 & 24.0 & 89 & 6 & 48 & 11 \\
\hline A2666-1.c1 & 21.5 & 94.5 & 38.8 & 76.1 & 38.9 & 24.5 & 848 & 115 & 712 & 121 \\
\hline A2666-1.c2 & 21.5 & 27.7 & 6.8 & 9.3 & 7.4 & 24.5 & 412 & 23 & 297 & 43 \\
\hline A2666-1.c3 & 21. & 57.1 & 5.1 & 24.8 & 7.6 & 25.0 & 128 & 13 & 59 & 26 \\
\hline $\mathrm{A} 2$ & 21.5 & 39.9 & 2.4 & 7.6 & 6.1 & 25.0 & 94 & 7 & 28 & 24 \\
\hline \multicolumn{11}{|c|}{ Run 0295.} \\
\hline$\overline{\mathrm{A} 53}$ & 22.0 & 80.8 & 24.4 & 63.1 & 24.7 & 24.5 & 1813 & 214 & 1539 & 233 \\
\hline A53s & 22.0 & 44.7 & 7.8 & 27.0 & 8.6 & 24.5 & 775 & 43 & 517 & 101 \\
\hline A539-1.c3 & 22.0 & 35.0 & 4.0 & 17.3 & 5.4 & 24.5 & 520 & 13 & 267 & 93 \\
\hline A539-1.c4 & 22.0 & 22.3 & 1.5 & 4.6 & 3.9 & 24.5 & 285 & 15 & 34 & 93 \\
\hline A539-2.c1 & 22.0 & 101.8 & 31.1 & 80.2 & 31.2 & 24.5 & 2217 & 76 & 1954 & 118 \\
\hline A539-2.c2 & 22.0 & 92.5 & 13.5 & 70.9 & 13.7 & 24.5 & 1055 & 26 & 802 & 94 \\
\hline A539-2.c3 & 22.0 & 44.9 & 4.6 & 23.3 & & 24.5 & 562 & 8 & 315 & 92 \\
\hline $2 . c 4$ & 22 & & & 2.5 & 3.0 & & & & 82 & 92 \\
\hline A569-1.c1 & 21.5 & 16.1 & 9.4 & 4.7 & 9.6 & 24.0 & 336 & 31 & 260 & 38 \\
\hline A569-1.c2 & 21.5 & 68.2 & 11.0 & 45.1 & 11.2 & 24.5 & 94 & 7 & 53 & 15 \\
\hline A569-1.c3 & 21.5 & 35.3 & 3.6 & 12.2 & & 24.5 & 49 & 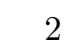 & 12 & 13 \\
\hline A569-1.c4 & 21.5 & 24.5 & 1.6 & 1.4 & 2.8 & 24.5 & 44 & 2 & 8 & 13 \\
\hline A634-1.c1 & 22.0 & 145.8 & 32.4 & 128.0 & 32.4 & 24.5 & 2282 & 118 & 1807 & 145 \\
\hline A634-1.c2 & 22.0 & & 9.1 & 30.3 & 9.2 & 24.5 & 1023 & 62 & 591 & 104 \\
\hline A634-1.c3 & 22.0 & & 4.2 & 10.1 & & 25.0 & 318 & 10 & 54 & 53 \\
\hline A634-1.c4 & 22.0 & 38.4 & 2.0 & 1.8 & 2.7 & 25.0 & 237 & 13 & -25 & 54 \\
\hline A779-1.c1 & 21.5 & 86.6 & 36.2 & 68.1 & 36.4 & 24.5 & 1083 & 59 & 940 & 65 \\
\hline A779-1.c2 & 21.5 & 113.7 & 14.8 & 78.2 & 15.6 & 25.0 & 309 & 26 & 227 & 31 \\
\hline A779-1.c3 & 21.5 & 91.2 & 6.3 & 55.7 & 7.9 & 25.0 & 188 & 7 & 118 & 16 \\
\hline A779-1.c4 & 21.5 & 45.3 & 2.2 & 9.8 & 5.3 & 25.0 & 112 & 8 & 47 & 16 \\
\hline A999-1.c1 & 22.0 & 98.5 & 36.6 & 68.2 & 36.6 & 25.0 & 1068 & 46 & 775 & 81 \\
\hline A999-1.c2 & 22.0 & 60.1 & 10.6 & 29.8 & 10.7 & 25.0 & 512 & 31 & 228 & 74 \\
\hline A999-1.c3 & 22.0 & 35.7 & 4.2 & 5.4 & 4.5 & 25.0 & 345 & 25 & 67 & 71 \\
\hline A999-1.c4 & 22.0 & 54.6 & 2.3 & 3.9 & 3.8 & 25.5 & 133 & 8 & -40 & 42 \\
\hline A1016-1.c1 & 22.0 & 119.2 & 30.9 & 92.5 & 30.9 & 25.0 & 685 & 29 & 451 & 58 \\
\hline A1016-1.c2 & 22.0 & 88.4 & 12.1 & 42.6 & 12.4 & 25.5 & 226 & 11 & 84 & 33 \\
\hline
\end{tabular}


Table 5-Continued

\begin{tabular}{|c|c|c|c|c|c|c|c|c|c|c|}
\hline Galaxy.reg & $m_{b}$ & $N_{p s}$ & \pm & $N_{G C}$ & \pm & $m_{c}$ & $P_{0}$ & \pm & $P_{G C}$ & \pm \\
\hline A1016-1.c3 & 22.0 & 53.1 & 4.9 & 7.3 & 5.7 & 25.5 & 153 & 5 & 15 & 31 \\
\hline A1016-1.c4 & 22.0 & 49.8 & 2.5 & 4.0 & 3.8 & 25.5 & 111 & . & -26 & 31 \\
\hline A1177-1.c2 & 22.0 & 34.1 & 8.3 & 18.5 & 8.5 & 24.5 & 1796 & 94 & 1158 & 150 \\
\hline A1177-1.c3 & 22.0 & 86.4 & 5.9 & 30.9 & 6.3 & 25.5 & 384 & 7 & 137 & 46 \\
\hline A1177-1.c4 & 22.0 & 58.3 & 2.3 & 2.8 & 3.2 & 25.5 & 251 & 13 & 8 & 47 \\
\hline A1367-1.c1 & 21.5 & 201.3 & 38.3 & 185.6 & & 24.5 & 448 & 32 & 396 & 35 \\
\hline A1367-1.c2 & 21.5 & 103.5 & 11.9 & 87.8 & 12.0 & 24.5 & 236 & 0 & 194 & 15 \\
\hline A1367-1.c3 & 21.5 & 76.3 & 5.2 & 43.3 & 6.0 & 25.0 & 64 & 4 & 40 & 9 \\
\hline $\mathrm{A} 13$ & 21.5 & 9 & 2.2 & 14.9 & 3.7 & 25.0 & 38 & 1 & 16 & 8 \\
\hline \multicolumn{11}{|c|}{ Runs 0395 \& 0995.} \\
\hline A347-1.c1 & 21.5 & 226.0 & 36.5 & 199.3 & 36.7 & 24.5 & 302 & 21 & 261 & 22 \\
\hline A347-1.c2 & 21.5 & 104.3 & 11.7 & 77.6 & 12.4 & 24.5 & 120 & & 88 & 10 \\
\hline A347-1.c3 & 21.5 & 62.5 & 4.7 & 35.8 & 6.2 & 24.5 & 69 & & 40 & 7 \\
\hline A347-1.c4 & 21.5 & 36.8 & 1.9 & 10.1 & 4.5 & 24.5 & 41 & 2 & 13 & 7 \\
\hline A397-1.c1 & 22.0 & 100.1 & 26.0 & 77.5 & 26.1 & 25.0 & 1549 & 71 & 1288 & 87 \\
\hline A397-1.c2 & 22.0 & .3 & 9.7 & 38.7 & 9.9 & 25.0 & & & 653 & 88 \\
\hline A397-1.c3 & 22.0 & 29.2 & 3.3 & 6.6 & 3.8 & 25.0 & 548 & 23 & 324 & 53 \\
\hline A397-1.c4 & 22.0 & 24.0 & 1.4 & 1.4 & 2.3 & 25.0 & 331 & 16 & 111 & 50 \\
\hline A569-1.c1 & 21.5 & & 13.9 & 9.5 & & & & & 485 & 105 \\
\hline & 21.5 & & & 18.5 & & & & 22 & 202 & 44 \\
\hline A569-1.c3 & 21.5 & 21.6 & 2.7 & 4.7 & 3.6 & 24.0 & 175 & 20 & 68 & 42 \\
\hline A569-1.c4 & 21.5 & & 1.4 & 0.7 & 2.8 & 24.0 & 150 & 16 & 45 & 41 \\
\hline A1185-1.c1 & 22.0 & 126.8 & 51.2 & 99.0 & 51.3 & 25.0 & 5627 & 4 & 4921 & 367 \\
\hline 1.c2 & 22.0 & 62.1 & 9.9 & 34.3 & 10.2 & 25.0 & 2327 & 137 & 1683 & 184 \\
\hline A1185-1.c3 & 22.0 & 39.6 & 3.8 & 11.8 & 4.6 & 25.0 & 1377 & 33 & 755 & 127 \\
\hline A1185-1.c4 & 22.0 & 30.1 & 1.9 & 2.3 & 3.2 & 25.0 & & 54 & 274 & 134 \\
\hline A1314- & 22.0 & 84.6 & 28.8 & 57.1 & 28.9 & 25.0 & 2531 & 239 & 1898 & 263 \\
\hline A1314-1.c2 & 22.0 & 85.9 & 12.3 & 58.4 & 12.6 & 25.0 & 1439 & 123 & 838 & 164 \\
\hline A1314-1.c3 & 22.0 & 51.6 & 4.8 & 24.1 & 5.6 & 25.0 & 908 & 34 & 323 & 113 \\
\hline A1314-1.c4 & 21.5 & & 0.0 & 20.4 & 6.2 & 25.5 & 342 & 12 & -19 & 68 \\
\hline A1656-3.c1 & 21.5 & 152.5 & 30.4 & 127.5 & 30.5 & 24.5 & 2417 & 103 & 2099 & 136 \\
\hline A1656-3.c2 & 21.5 & 62.9 & 9.2 & 37.9 & 9.5 & 24.5 & 1323 & 96 & 1053 & 128 \\
\hline A1656-3.c3 & 21.5 & 36.1 & 3.4 & 11.1 & 4.2 & 24.5 & 600 & 22 & 347 & 88 \\
\hline A1656-3.c4 & 21.5 & 27.5 & 1.7 & 2.5 & 3.0 & 24.5 & 391 & 21 & 144 & 88 \\
\hline A2197-1.c2 & 22.0 & 39.3 & 8.0 & 21.0 & 9.0 & 24.5 & 2511 & 189 & 1406 & 430 \\
\hline A2197-1.c3 & 22.0 & 25.3 & 3.1 & 7.0 & 5.2 & 24.5 & 1318 & 126 & 252 & 406 \\
\hline A2197-1.c4 & 22.0 & 21.7 & 1.3 & 3.4 & 4.4 & 24.5 & 1128 & 58 & 73 & 390 \\
\hline A2197-2.c1 & 22.0 & 161.8 & 50.3 & 138.3 & 50.5 & 25.0 & 1451 & 61 & 1221 & 79 \\
\hline A2197-2.c2 & 22.0 & 81.7 & 12.7 & 58.2 & 13.6 & 25.0 & 857 & 56 & 653 & 74 \\
\hline A2197-2.c3 & 22.0 & 60.0 & 5.0 & 36.5 & 7.0 & 25.0 & 499 & 15 & 307 & 51 \\
\hline A2197-2.c4 & 22.0 & 32.0 & 1.7 & 8.5 & 5.2 & 25.0 & 281 & 14 & 94 & 51 \\
\hline A2162-1.c1 & 22.0 & 55.7 & 20.1 & 45.1 & 20.2 & 24.0 & 5886 & 539 & 5009 & 569 \\
\hline
\end{tabular}


Table 5-Continued

\begin{tabular}{lcrrrrrrrrr}
\hline \hline Galaxy.reg & $m_{b}$ & \multicolumn{1}{c}{$N_{p s}$} & \pm & $N_{G C}$ & \pm & $m_{c}$ & $P_{0}$ & \pm & $P_{G C}$ & \pm \\
\hline A2162-1.c2 & 22.0 & 71.3 & 12.1 & 50.9 & 12.3 & 24.5 & 1775 & 124 & 1240 & 168 \\
A2162-1.c3 & 22.0 & 37.4 & 3.8 & 17.0 & 4.4 & 24.5 & 835 & 40 & 312 & 120 \\
A2162-1.c4 & 22.0 & 23.7 & 1.6 & 3.3 & 2.7 & 24.5 & 616 & 34 & 96 & 118 \\
A2634-1.c1 & 22.0 & 110.3 & 30.4 & 91.5 & 30.7 & 24.5 & 2435 & 158 & 2162 & 173 \\
A2634-1.c2 & 22.0 & 75.6 & 10.5 & 56.8 & 11.3 & 24.5 & 1207 & 78 & 960 & 104 \\
A2634-1.c3 & 22.0 & 47.2 & 4.2 & 28.4 & 5.9 & 24.5 & 738 & 37 & 501 & 77 \\
A2634-1.c4 & 22.0 & 24.2 & 1.6 & 5.4 & 4.4 & 24.5 & 429 & 13 & 195 & 69 \\
\hline
\end{tabular}

Table 6. Metric Specific Frequencies and GCLF Widths in CMB Frame

\begin{tabular}{|c|c|c|c|c|c|c|c|}
\hline Galaxy & $M_{V}$ & $S_{N}+$ & $\sigma_{\mathrm{LF}}+$ & Galaxy & $M_{V}$ & $S_{N}{ }_{-}^{+}$ & $\sigma_{\mathrm{LF}}+$ \\
\hline A262-1 & -22.08 & $5.0_{1.3}^{1.5}$ & 1.38 .14 & A1314-1 & -22.20 & $4.2_{1.0}^{1.1}$ & 1.58 .21 \\
\hline A347-1 & -21.86 & $\begin{array}{l}5.81 .6 \\
1.3\end{array}$ & 1.36 .10 & A1367-1 & -22.29 & 5.31 .4 & 1.53 .11 \\
\hline A397-1 & -22.02 & $4.7 \begin{array}{l}1.4 \\
1.1\end{array}$ & 1.43 .11 & A1656-1 & -22.75 & 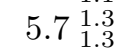 & 1.37 .12 \\
\hline A539-1 & -21.73 & $9.1_{2.6}^{3.0}$ & $1.42 . .13$ & A1656-2 & -22.55 & $9.3_{2.0}^{2.0}$ & $\begin{array}{l}1.43 .09 \\
.09\end{array}$ \\
\hline A539-2 & -22.16 & $9.4 \begin{array}{l}3.0 \\
2.4\end{array}$ & 1.46 .12 & A1656-3 & -22.16 & $4.6_{1.3}^{1.5}$ & $\begin{array}{l}1.33 .11 \\
.11\end{array}$ \\
\hline A569-1 & -21.59 & $3.0 \begin{array}{l}1.2 \\
1.0\end{array}$ & 1.42 .24 & A2162-1 & -21.98 & $7.4_{1.8}^{2.2}$ & 1.73 .16 \\
\hline A634-1 & -21.65 & $4.0 \begin{array}{l}1.2 \\
1.0\end{array}$ & 1.76 .18 & A2197-1 & -22.16 & 2.51 .4 & 1.55 .31 \\
\hline A779-1 & -22.56 & $4.1 \begin{array}{l}1.0 \\
0.9\end{array}$ & 1.34 .12 & A2197-2 & -22.22 & 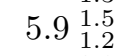 & 1.49 .11 \\
\hline A999-1 & -21.55 & 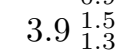 & 1.56 .24 & A2199-1 & -22.44 & $8.1_{1.9}^{2.3}$ & $\begin{array}{l}1.53 .10 \\
.09\end{array}$ \\
\hline A1016-1 & -21.33 & $3.3 \begin{array}{l}1.2 \\
1.1\end{array}$ & 1.83 .31 & A2634-1 & -22.40 & $7.5_{1.7}^{2.1}$ & 1.51 .09 \\
\hline A1177-1 & -22.00 & $4.2_{1.0}^{1.3}$ & 1.49 .18 & A2666-1 & -22.24 & $3.5_{1.0}^{1.1}$ & 1.43 .24 \\
\hline A1185-1 & -22.03 & $6.4_{1.4}^{1.8}$ & 1.33 .10 & & & & \\
\hline
\end{tabular}

Note. - Columns list: galaxy name; absolute $V$ magnitude of the region of the galaxy in which the GC measurements were made (assumes $H_{0}=80$ and CMB velocities); $S_{N}$ calculated within $40 \mathrm{kpc}$ assuming $\sigma=1.40 \pm 0.05$ for the GCLF width; actual measured value of $\sigma$. 
Table 7. Comparison of $S_{N}^{f l u}$ and $S_{N}^{c n t}$ for $\sigma=1.40 \mathrm{mag}$

\begin{tabular}{rrrrrrrrrrrrrr}
\hline \hline Galaxy & $S_{N}^{\text {flu }}$ & \pm & \multicolumn{1}{c}{$S_{N}^{\text {cnt }}$} & \pm & \multicolumn{1}{c}{$\sigma_{\text {dev }}$} & Galaxy & $S_{N}^{\text {flu }}$ & \pm & $S_{N}^{\text {cnt }}$ & \pm & $\sigma_{\text {dev }}$ \\
\hline $\mathrm{A} 262-1$ & 5.6 & 1.6 & 4.7 & 1.1 & 0.50 & $\mathrm{~A} 1185-1$ & 6.7 & 0.7 & 5.4 & 1.3 & 0.96 \\
$\mathrm{~A} 347-1$ & 6.8 & 1.3 & 5.0 & 1.1 & 1.18 & $\mathrm{~A} 1314-1$ & 3.4 & 0.7 & 6.7 & 1.1 & 2.65 \\
$\mathrm{~A} 397-1$ & 5.3 & 0.6 & 3.6 & 0.9 & 1.75 & $\mathrm{~A} 1367-1$ & 5.6 & 1.0 & 6.0 & 0.6 & 0.35 \\
$\mathrm{~A} 539-1$ & 8.2 & 1.9 & 10.3 & 2.4 & 0.69 & $\mathrm{~A} 1656-3$ & 5.7 & 1.1 & 3.8 & 1.0 & 1.29 \\
$\mathrm{~A} 539-2$ & 8.7 & 1.5 & 11.0 & 1.7 & 1.00 & $\mathrm{~A} 2162-1$ & 6.3 & 1.1 & 15.5 & 2.8 & 3.17 \\
$\mathrm{~A} 569-1$ & 3.9 & 2.1 & 2.7 & 1.5 & 0.46 & $\mathrm{~A} 2197-1$ & 1.8 & 1.5 & 3.7 & 1.9 & 0.80 \\
$\mathrm{~A} 634-1$ & 2.7 & 1.2 & 6.6 & 1.3 & 2.28 & $\mathrm{~A} 2197-2$ & 5.5 & 0.7 & 7.1 & 1.1 & 1.22 \\
$\mathrm{~A} 779-1$ & 4.4 & 0.6 & 3.7 & 0.6 & 0.96 & $\mathrm{~A} 2199-1$ & 7.5 & 0.8 & 11.2 & 1.4 & 2.37 \\
$\mathrm{~A} 999-1$ & 3.5 & 1.7 & 4.8 & 1.7 & 0.57 & $\mathrm{~A} 2634-1$ & 7.1 & 0.8 & 9.4 & 1.5 & 1.43 \\
$\mathrm{~A} 1016-1$ & 2.6 & 1.7 & 5.0 & 1.3 & 1.14 & $\mathrm{~A} 2666-1$ & 3.7 & 1.0 & 3.0 & 0.9 & 0.53 \\
$\mathrm{~A} 1177-1$ & 3.9 & 0.8 & 4.7 & 1.1 & 0.64 & & & & & & \\
\hline
\end{tabular}

Table 8. Metric Specific Frequencies and GCLF Widths in ACI Frame

\begin{tabular}{|c|c|c|c|c|c|c|c|}
\hline Galaxy & $M_{V}$ & $S_{N}^{+}$ & $\sigma_{\mathrm{LF}}{ }_{-}^{+}$ & Galaxy & $M_{V}$ & $S_{N}^{+}$ & $\sigma_{\mathrm{LF}}{ }_{-}^{+}$ \\
\hline A262-1 & -22.56 & $5.4_{1.5}^{1.8}$ & 1.53 .14 & A1314-1 & -22.32 & $4.3_{1.0}^{1.2}$ & 1.62 .21 \\
\hline A347-1 & -22.28 & $6.3_{1.5}^{1.8}$ & 1.48 .10 & A1367-1 & -22.28 & $5.3 \begin{array}{l}1.4 \\
1.1\end{array}$ & 1.53 .11 \\
\hline A397-1 & -22.39 & $5.2 \begin{array}{l}1.7 \\
1.3\end{array}$ & 1.53 .110 & A1656-1 & -22.75 & $5.7_{1.3}^{1.3}$ & 1.37 .12 \\
\hline A539-1 & -22.07 & $10.1_{2.9}^{3.5}$ & 1.51 .13 & A1656-2 & -22.55 & $9.3_{2.0}^{2.0}$ & $\begin{array}{l}1.43 .09 \\
.09\end{array}$ \\
\hline A539-2 & -22.50 & $10.5_{2.8}^{3.5}$ & 1.55 .12 & A1656-3 & -22.16 & $4.6_{1.3}^{1.5}$ & 1.33 .11 \\
\hline A569-1 & -21.87 & $\begin{array}{l}3.31 .2 \\
1.0\end{array}$ & 1.50 .24 & A2162-1 & -22.01 & $\begin{array}{l}7.5_{1.9}^{2.3} \\
1.9\end{array}$ & 1.74 .16 \\
\hline A634-1 & -21.86 & $4.1_{1.1}^{1.3}$ & 1.81 .18 & A2197-1 & -22.21 & $2.6 \begin{array}{l}1.4 \\
1.3\end{array}$ & 1.56 .30 \\
\hline A779-1 & -22.70 & $\begin{array}{l}4.11 .0 \\
0.9\end{array}$ & 1.39 .12 & A2197-2 & -22.28 & $\begin{array}{ll}5.9 & 1.5 \\
1.2\end{array}$ & 1.51 .11 \\
\hline A999-1 & -21.67 & $4.0 \begin{array}{l}1.6 \\
1.4\end{array}$ & 1.59 .24 & A2199-1 & -22.50 & $8.2_{1.9}^{2.4}$ & $\begin{array}{r}1.55 .09 \\
.09\end{array}$ \\
\hline A1016-1 & -21.45 & $3.3_{1.1}^{1.2}$ & 1.86 .231 & A2634-1 & -22.72 & $8.1_{1.9}^{2.4}$ & 1.61 .10 \\
\hline A1177-1 & -22.08 & $4.3_{1.1}^{1.3}$ & 1.51 .18 & A2666-1 & -22.58 & $3.8_{1.1}^{1.3}$ & 1.53 .23 \\
\hline A1185-1 & -22.11 & $6.5_{1.5}^{1.9}$ & 1.35 .10 & & & & \\
\hline
\end{tabular}

Note. - Same as Table 6 but all calculations done in the ACI frame. 
Table 9: Neighboring Galaxy Counts

\begin{tabular}{r|rrr|rr}
\hline \hline$L$ & \multicolumn{3}{|c|}{$.10 L^{*}$} & \multicolumn{2}{|c}{$.05 L^{*}$} \\
$r<$ & 32 & 40 & 50 & 32 & $40 \quad\left(h^{-1} \mathrm{kpc}\right)$ \\
Galaxy & $N_{n}$ & $N_{n}$ & $N_{n}$ & $N_{n}$ & $N_{n}$ \\
\hline $\mathrm{A} 262-1$ & 3 & 5 & $\geq 5$ & 5 & 7 \\
$\mathrm{~A} 347-1$ & 0 & 0 & $\geq 0$ & 0 & 0 \\
$\mathrm{~A} 397-1$ & 2 & 2 & 2 & 4 & 4 \\
$\mathrm{~A} 539-1$ & 2 & 4 & 4 & 2 & 4 \\
$\mathrm{~A} 539-2$ & 4 & 6 & 7 & 6 & 8 \\
$\mathrm{~A} 569-1$ & 0 & 1 & 3 & 0 & 1 \\
$\mathrm{~A} 634-1$ & 0 & 1 & 1 & 0 & 1 \\
$\mathrm{~A} 779-1$ & 2 & 3 & 3 & 3 & 4 \\
$\mathrm{~A} 999-1$ & 2 & 4 & 4 & 3 & 6 \\
$\mathrm{~A} 1016-1$ & 1 & 2 & 2 & 1 & 2 \\
$\mathrm{~A} 1177-1$ & 0 & 2 & 2 & 1 & 4 \\
$\mathrm{~A} 1185-1$ & 3 & 3 & 3 & 4 & 5 \\
$\mathrm{~A} 1314-1$ & 0 & 1 & 3 & 1 & 2 \\
$\mathrm{~A} 1367-1$ & 3 & 3 & 3 & 4 & 5 \\
$\mathrm{~A} 1656-1$ & 2 & 4 & 6 & 3 & 5 \\
$\mathrm{~A} 1656-2$ & 5 & 7 & 11 & 5 & 7 \\
$\mathrm{~A} 1656-3$ & 1 & 1 & 2 & 1 & 1 \\
$\mathrm{~A} 2162-1$ & 0 & 1 & 1 & 0 & 3 \\
$\mathrm{~A} 2197-1$ & 0 & 1 & 1 & 0 & 1 \\
$\mathrm{~A} 2197-2$ & 1 & 1 & 2 & 2 & 2 \\
$\mathrm{~A} 2199-1$ & 5 & 5 & 8 & 6 & 7 \\
$\mathrm{~A} 2634-1$ & 2 & 3 & 3 & 3 & 4 \\
$\mathrm{~A} 2666-1$ & 1 & 1 & 3 & 1 & 1 \\
\hline
\end{tabular}




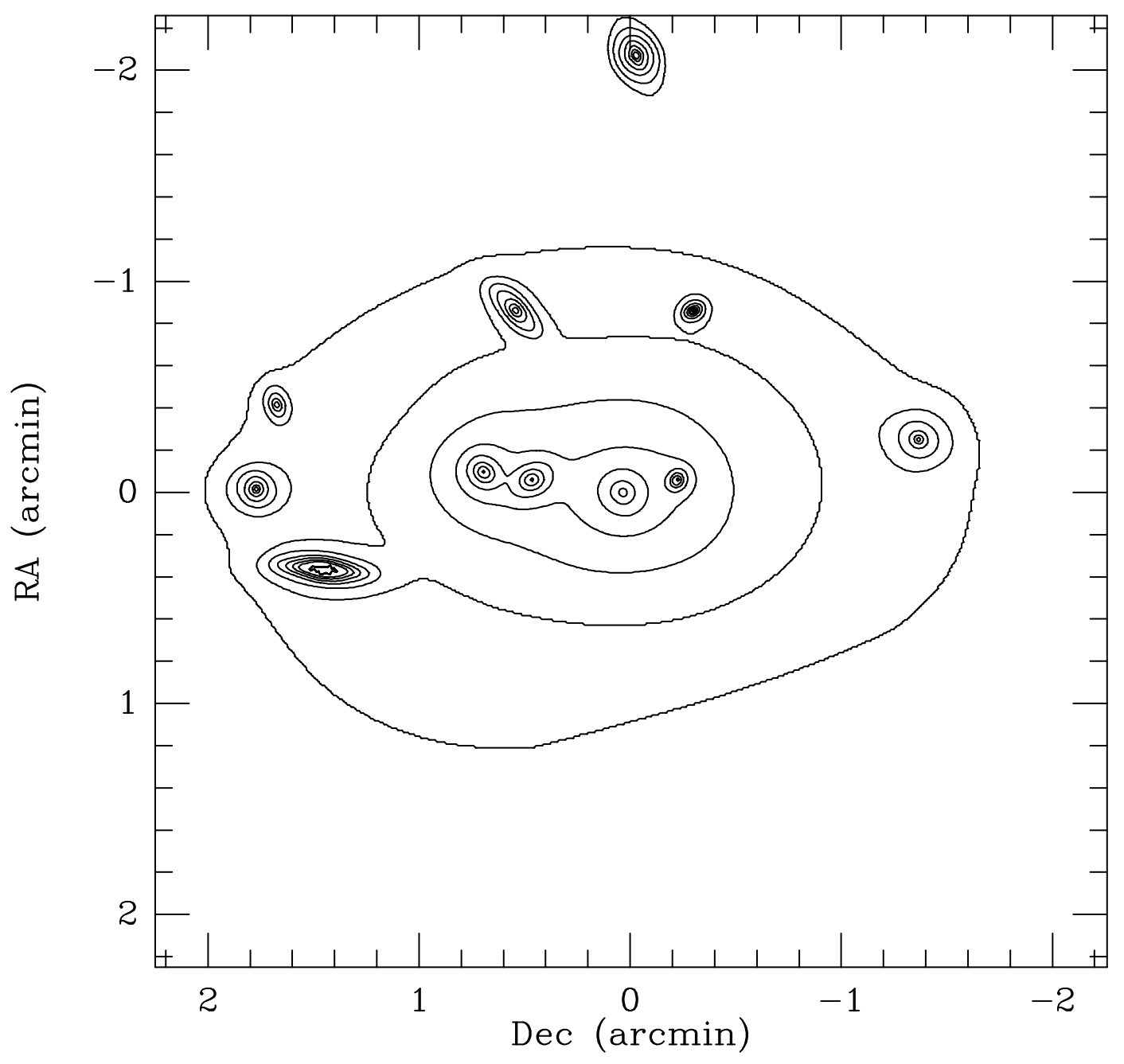

Fig. 1. - The isophotal model for the complex A539-2 galaxy system, generated by the iterative fitting procedure described in the text. The isophotal contours are plotted in increments of 1 mag, with the outermost contour at $R=24.5 \mathrm{mag} / \operatorname{arcsec}^{2}$. 


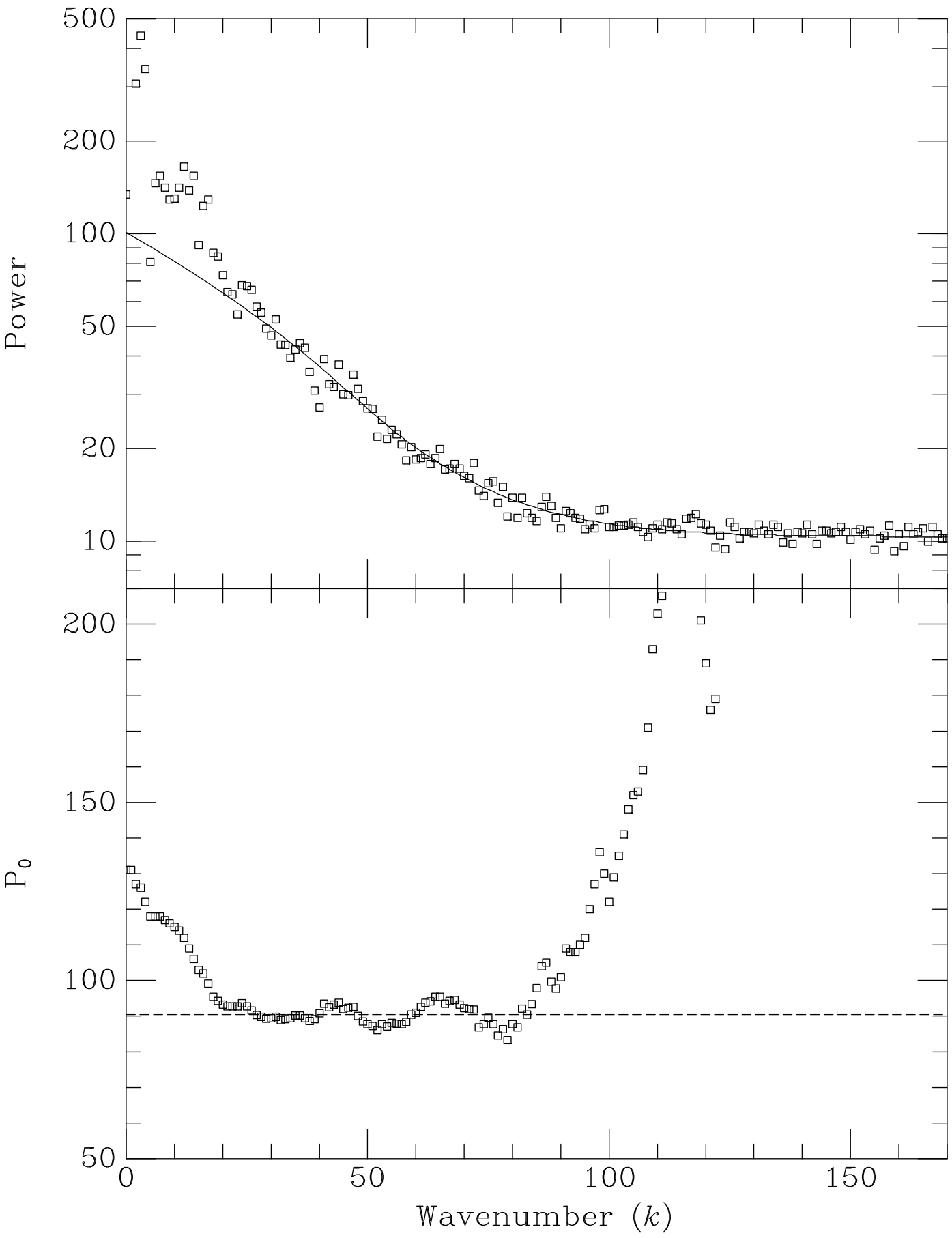

Fig. 2.- Power spectrum of the A2197-2 residual image for an annulus extending from 128 to 256 pixels in radius $(a)$, and the power spectrum normalization $P_{0}$ as a function of the starting wavenumber of the fit $(b)$. The solid curve is an example fit to the power spectrum, and the dashed line is the final value chosen for $P_{0}$. 


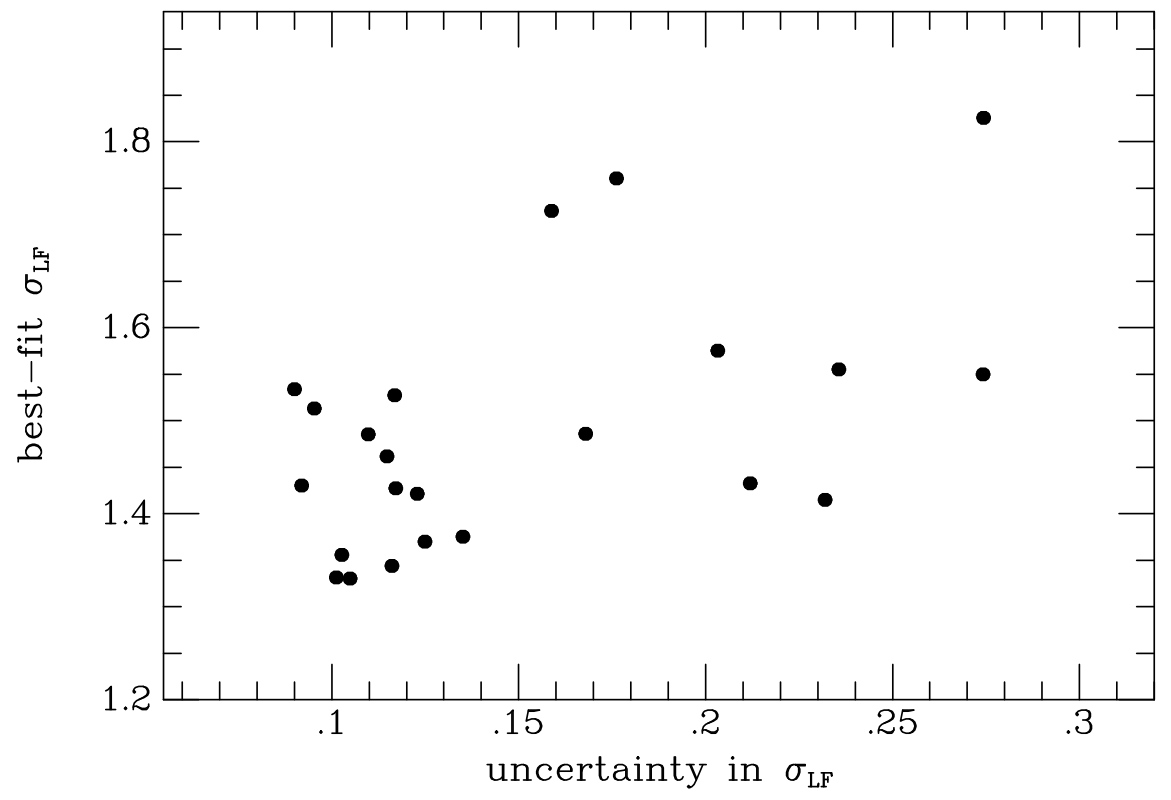

Fig. 3.- The derived GCLF width $\sigma$ in the CMB frame is plotted against its uncertainty, showing that the more uncertain values tend to be biased high. See text for details.

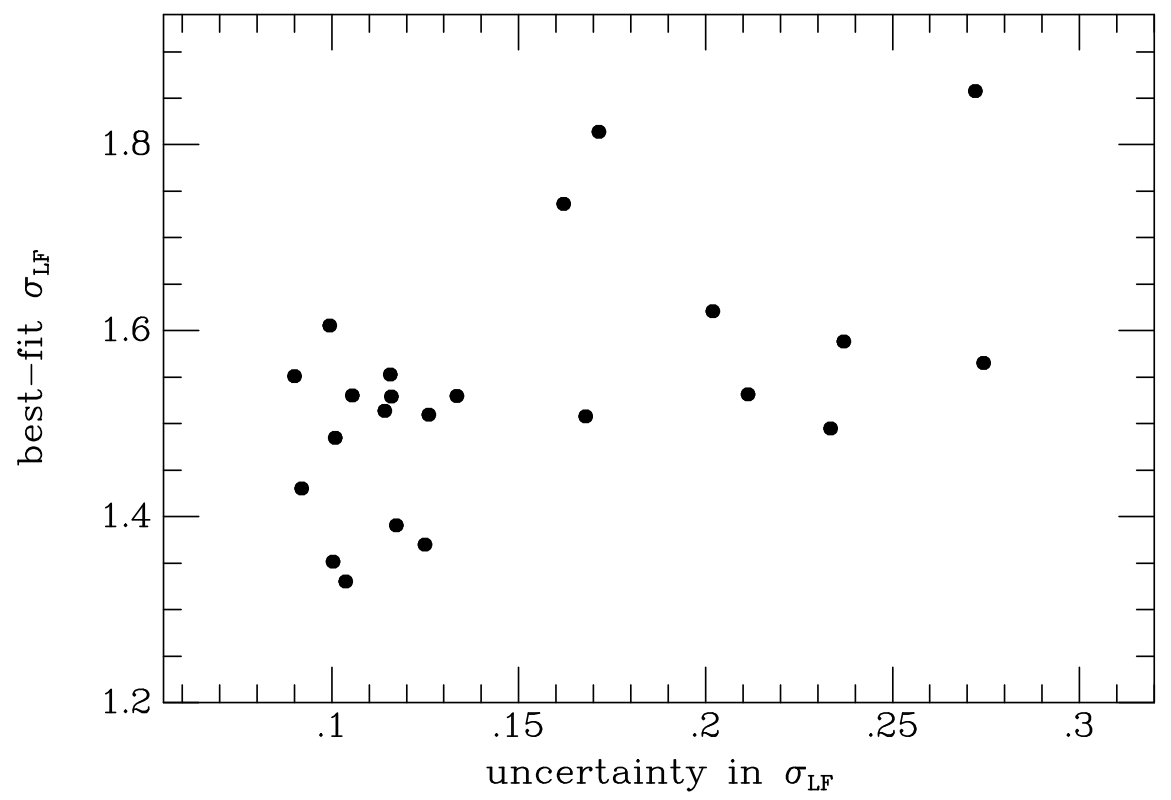

Fig. 4. - The derived GCLF width $\sigma$ in the ACI frame is plotted against its uncertainty. Again, the more uncertain values tend to be high, but here the median of the well-determined values is also high compared to the CMB frame results. 

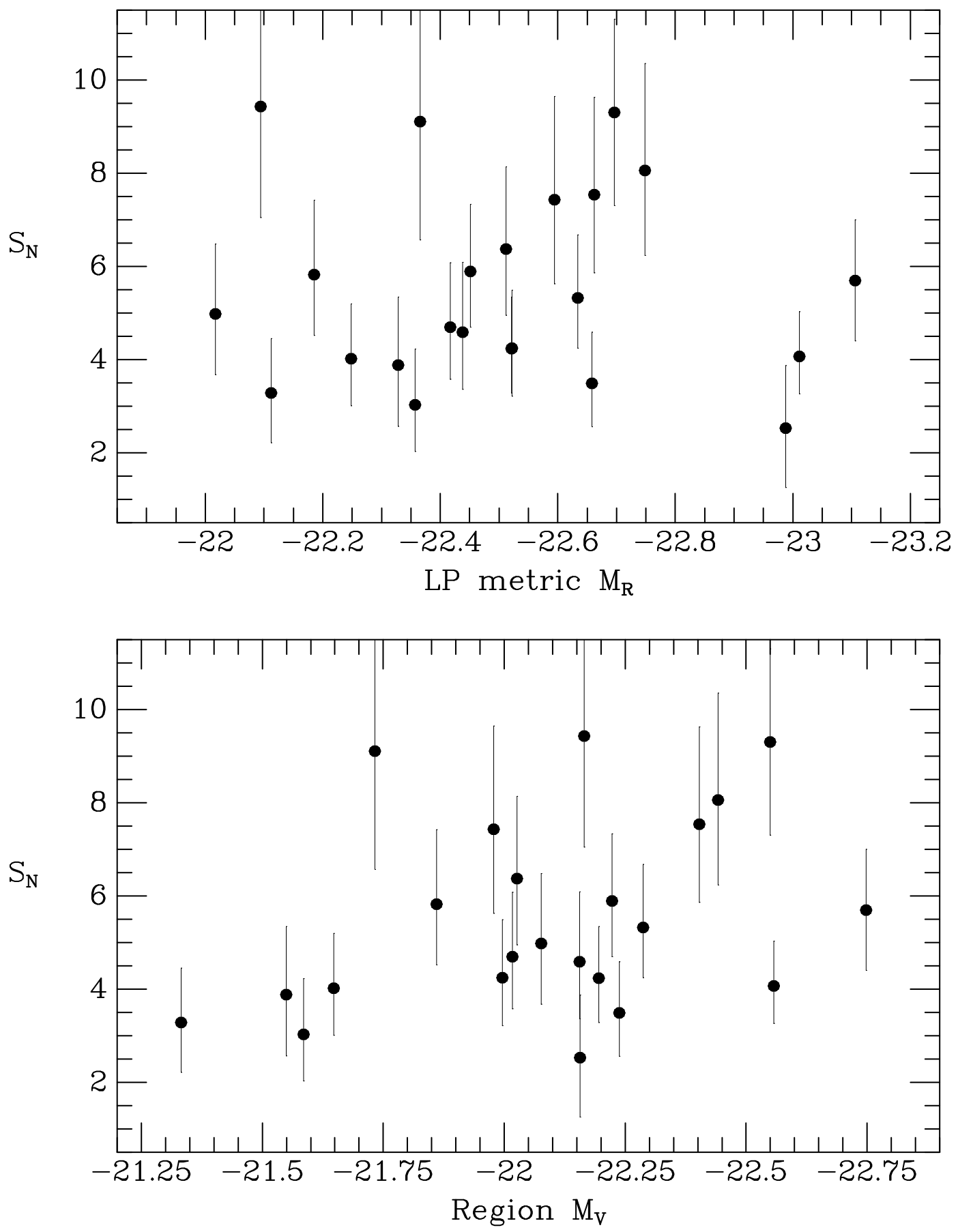

Fig. 5.- $S_{N}$ is plotted against (a) metric absolute magnitude from Lauer \& Postman (1994), and (b) total absolute magnitude of the specific region of each galaxy in which $S_{N}$ was measured (i.e., from $\sim 3$ to $32 h^{-1} \mathrm{kpc}$ ). All quantities are calculated in the CMB frame. 

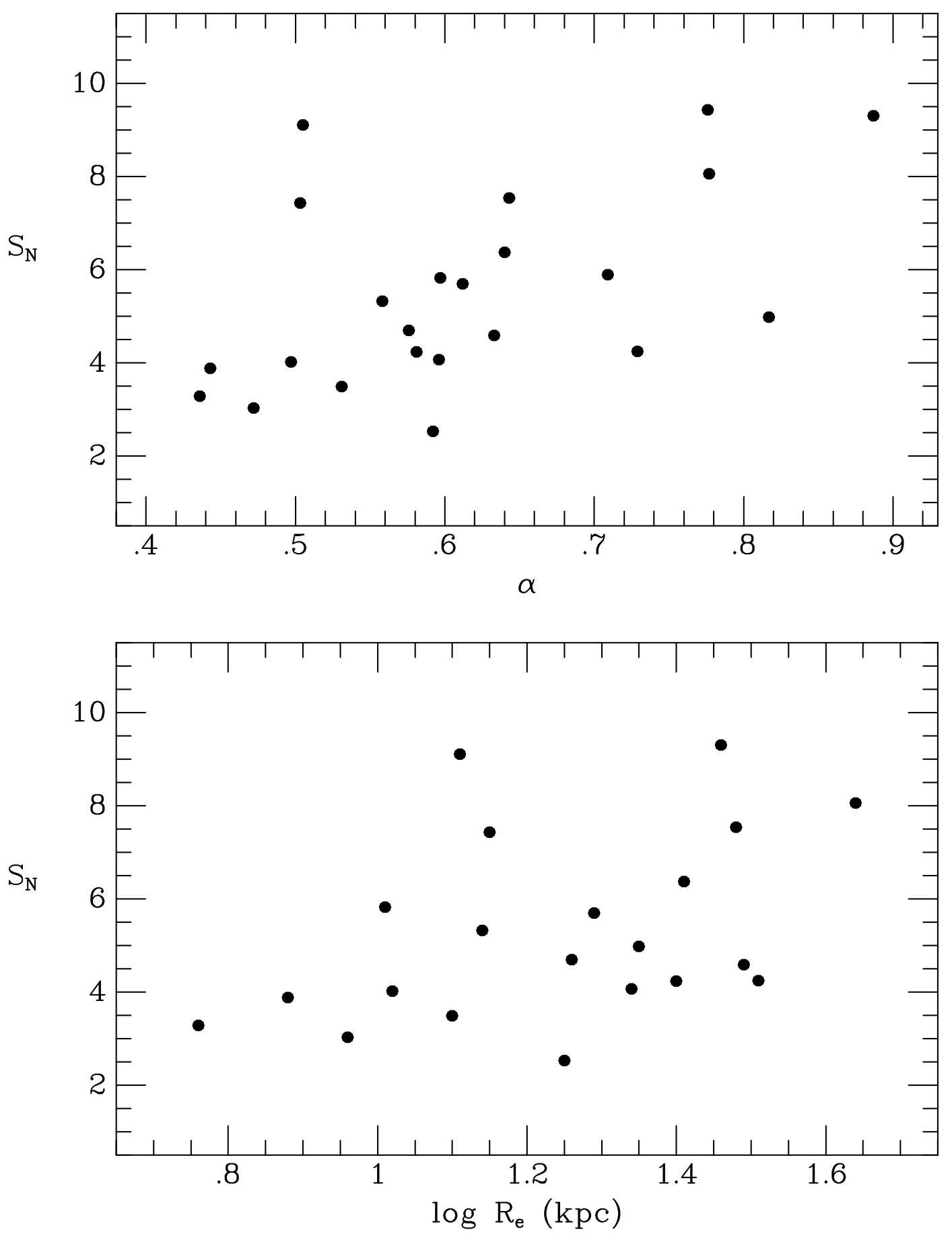

Fig. 6.- $S_{N}$ is plotted against two measures of the galaxy profile, $(a)$ the structure parameter $\alpha$ and $(b)$ the logarithm of the effective radius $R_{e}$. Errorbars have been omitted for the sake of clarity. 


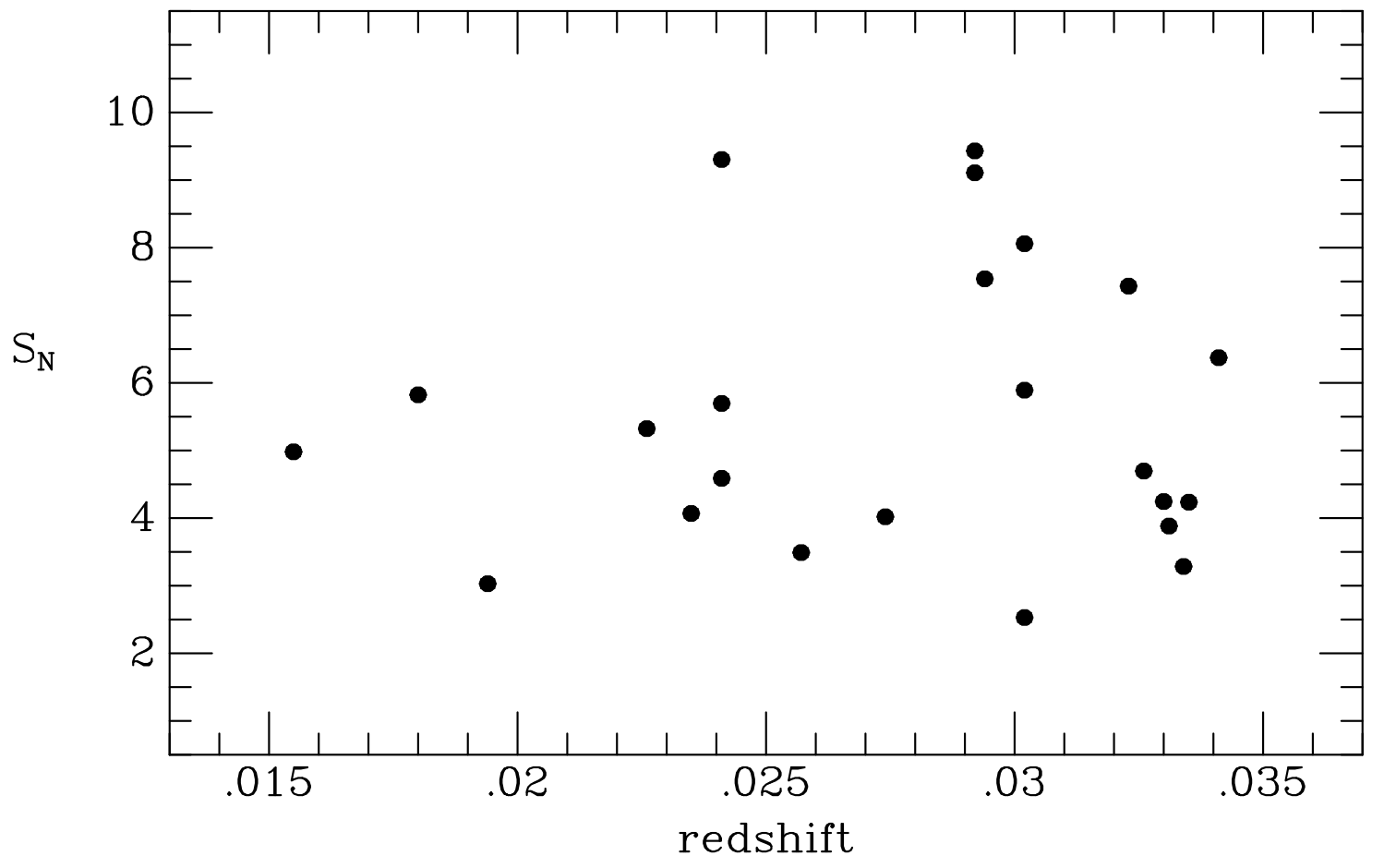

Fig. 7. - $S_{N}$ is plotted against the cluster redshift in the CMB frame. Though this is the redshift used in deriving the $S_{N}$ values, there is a gratifying lack of correlation. 

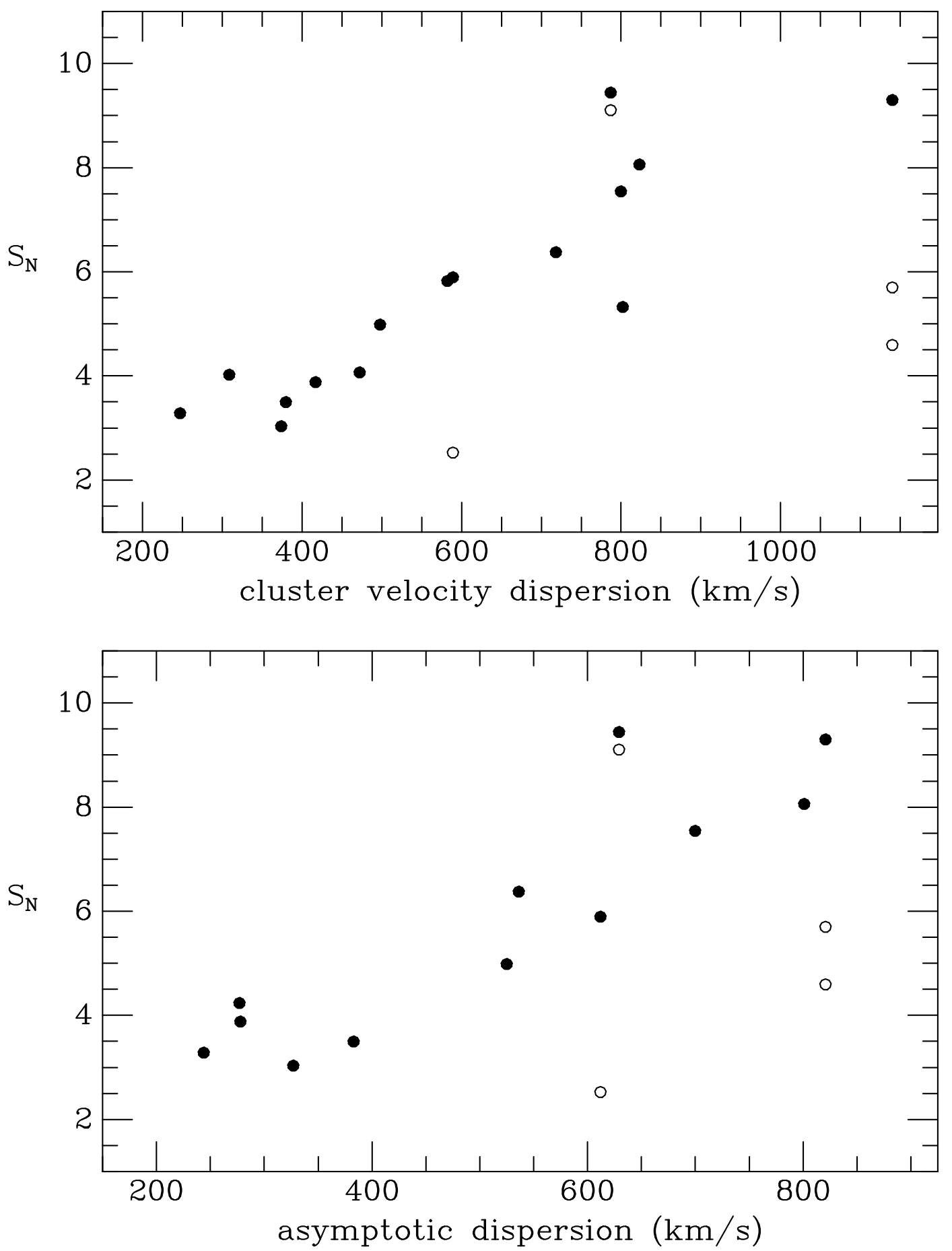

Fig. 8. - The correlation between $S_{N}$ and cluster central velocity dispersion. In $(a)$, central dispersions have been collected from various sources in the literature. In (b) the asymptotic velocity dispersions reported by Fadda et al. (1996) are used. Open symbols represent the "secondary" galaxies in clusters with more than one member in the present sample (see text for details); filled circles are rest of the sample. 


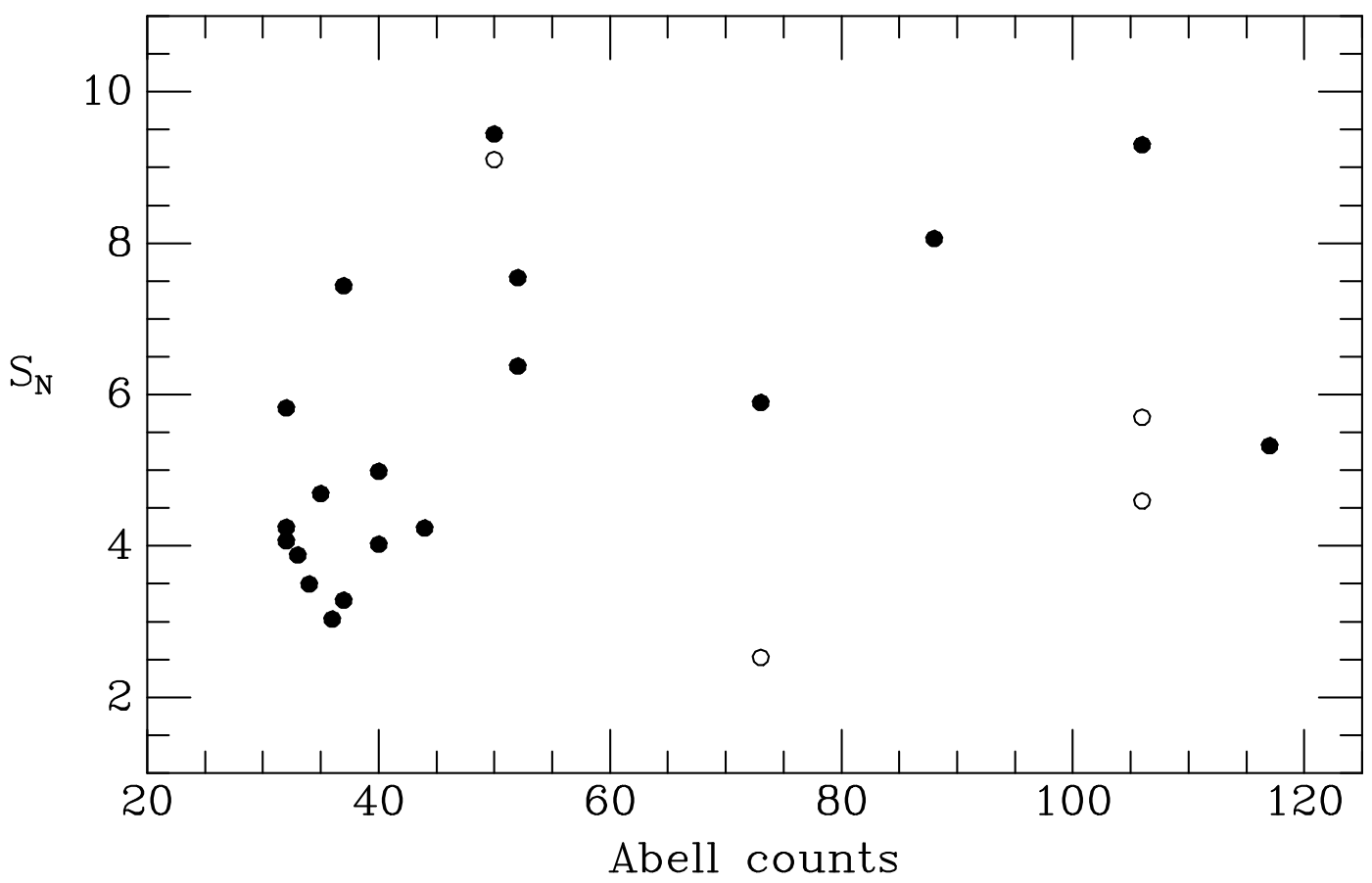

Fig. 9. $-S_{N}$ is plotted against Abell counts, a measure of overall cluster richness. Symbols are as in Figure 8 . 

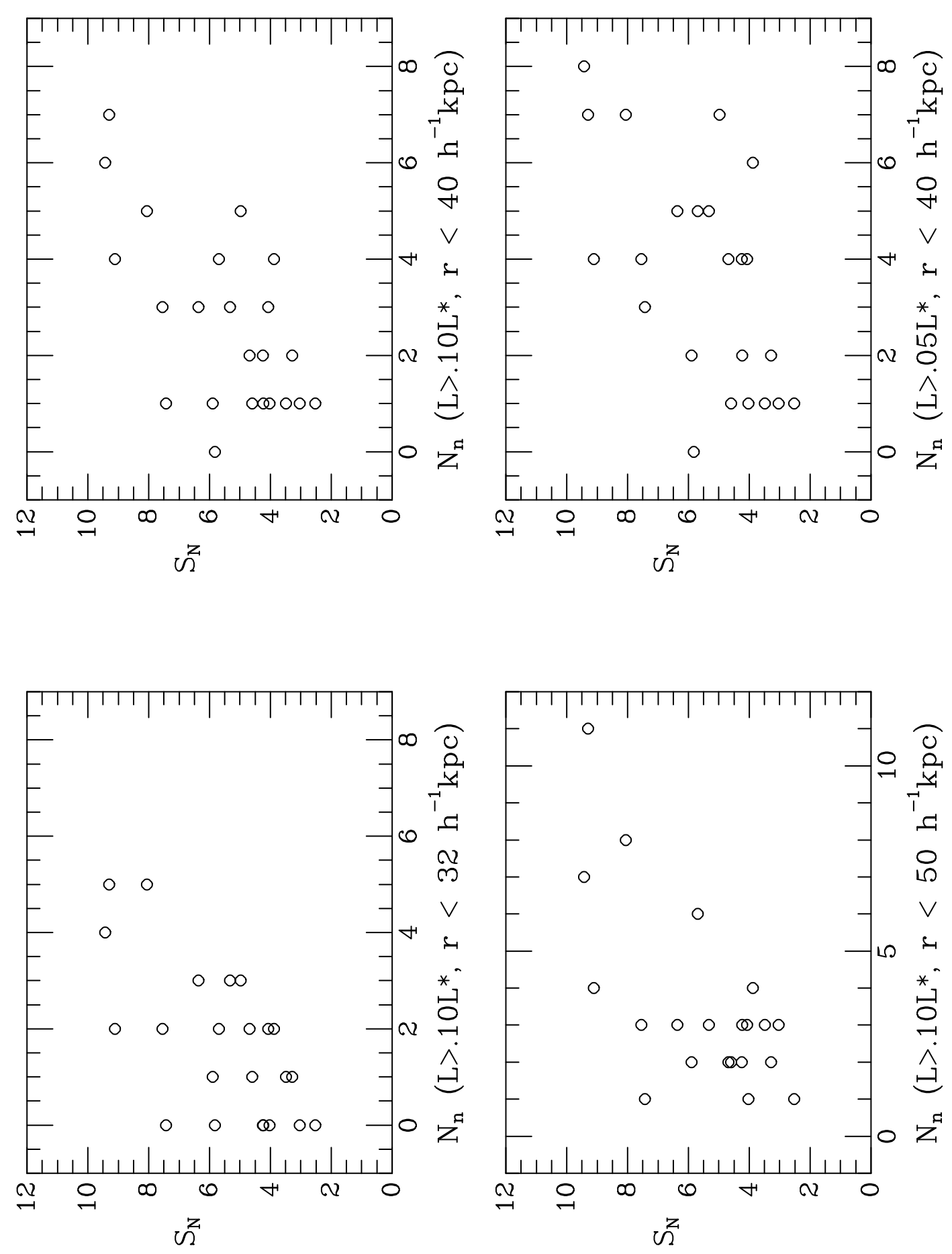

Fig. 10.- $S_{N}$ is plotted against the total number of neighboring galaxies brighter than $0.1 L^{*}$ within 32,40 , and $50 h^{-1} \mathrm{kpc}$ of the galaxy center, and against the number of neighbors brighter than $0.05 L^{*}$ within $40 h^{-1} \mathrm{kpc}$. 

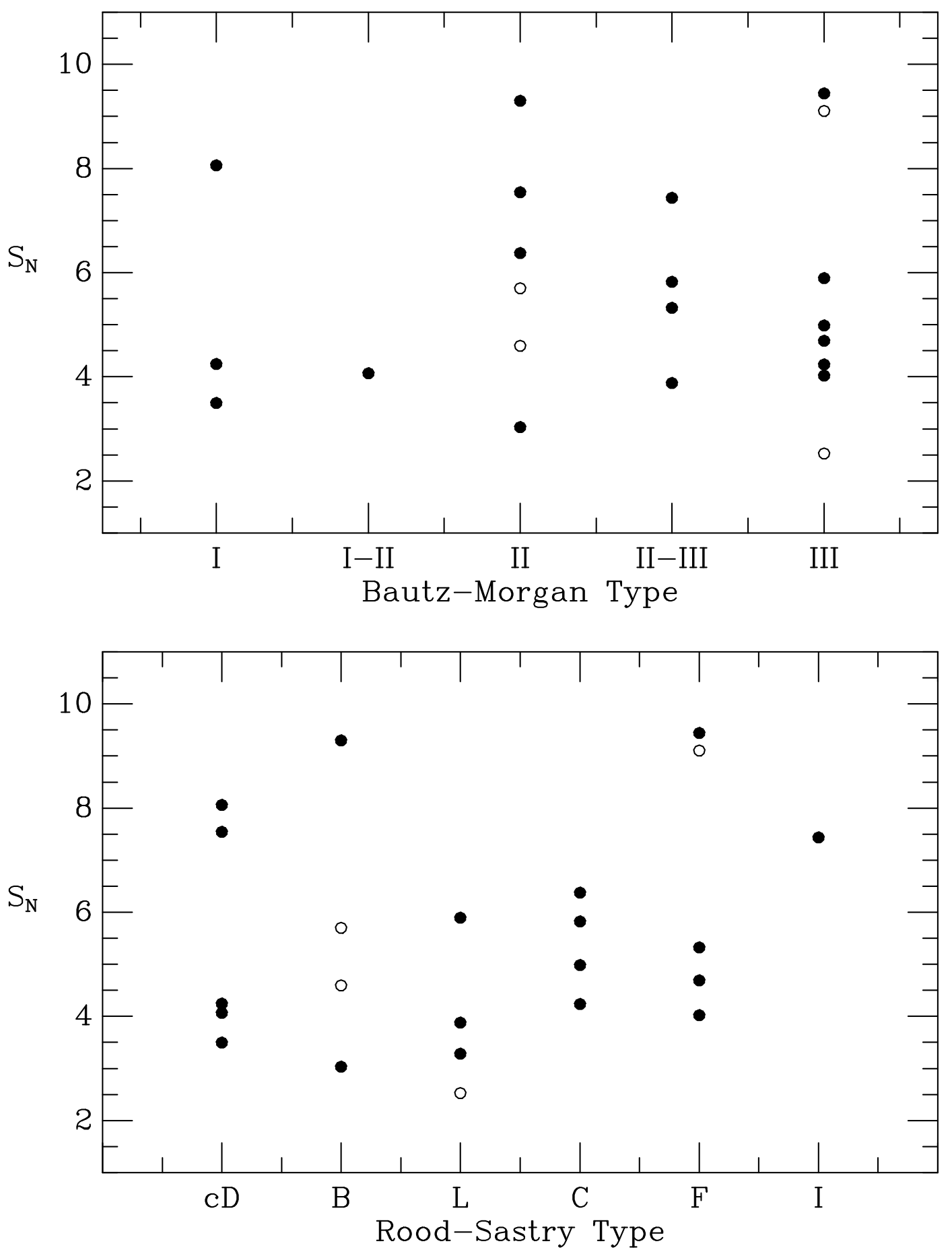

Fig. 11. - $S_{N}$ is plotted against morphological type in the Bautz-Morgan and Rood-Sastry classification systems. Unlike previous investigations based on many fewer clusters, we find no evidence of any correlation in these data. Here and in the following figures, symbols are as in Figure 8. 

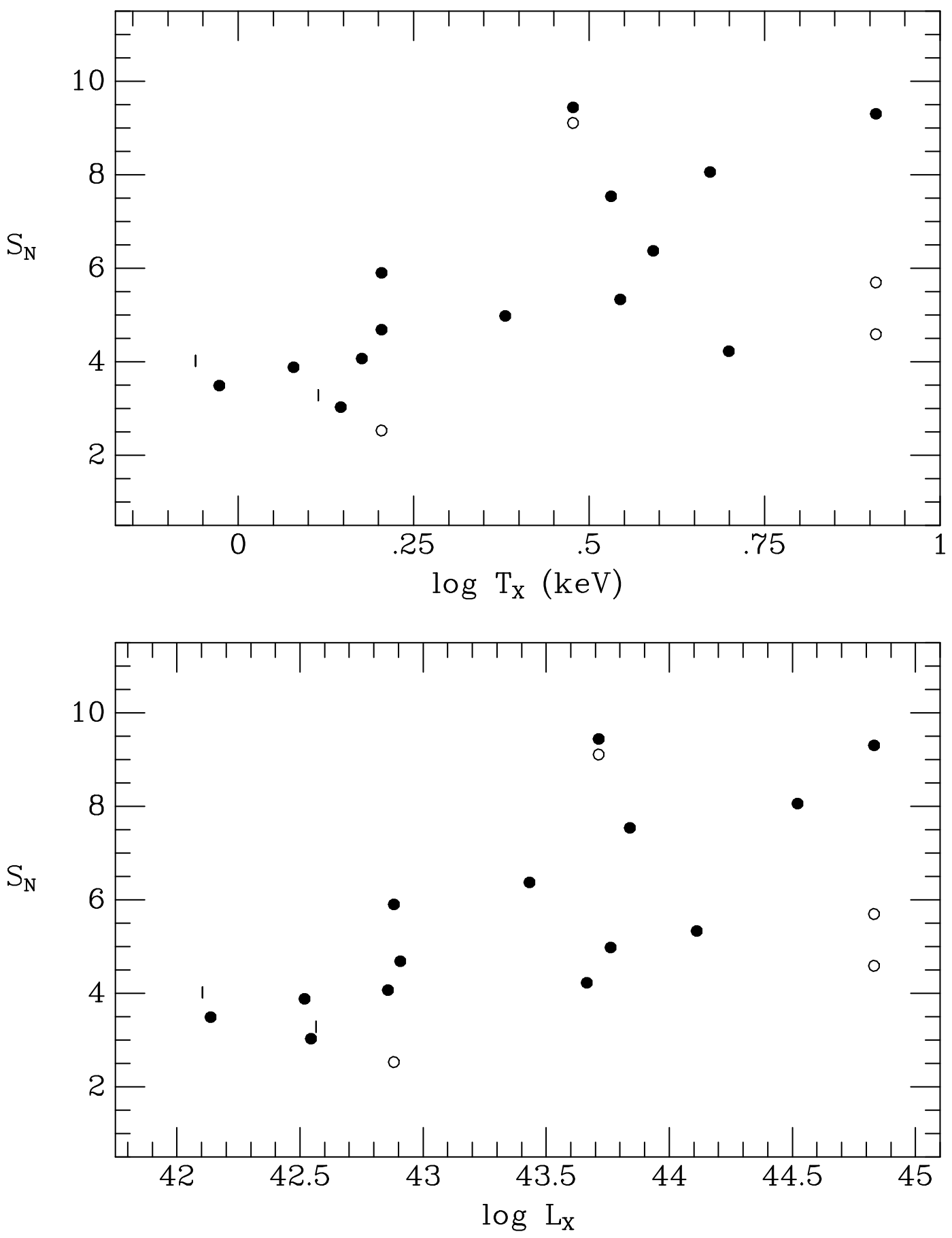

Fig. 12.- $S_{N}$ is plotted against $(a)$ temperature of the X-ray emitting intracluster gas, and $(b)$ total X-ray luminosity within $1 \mathrm{Mpc}$ of the cluster X-ray center (with $H_{0}=50$, as given by Jones \& Forman 1997). Short vertical lines represent galaxies in clusters with only upper limits on their X-ray emission. 


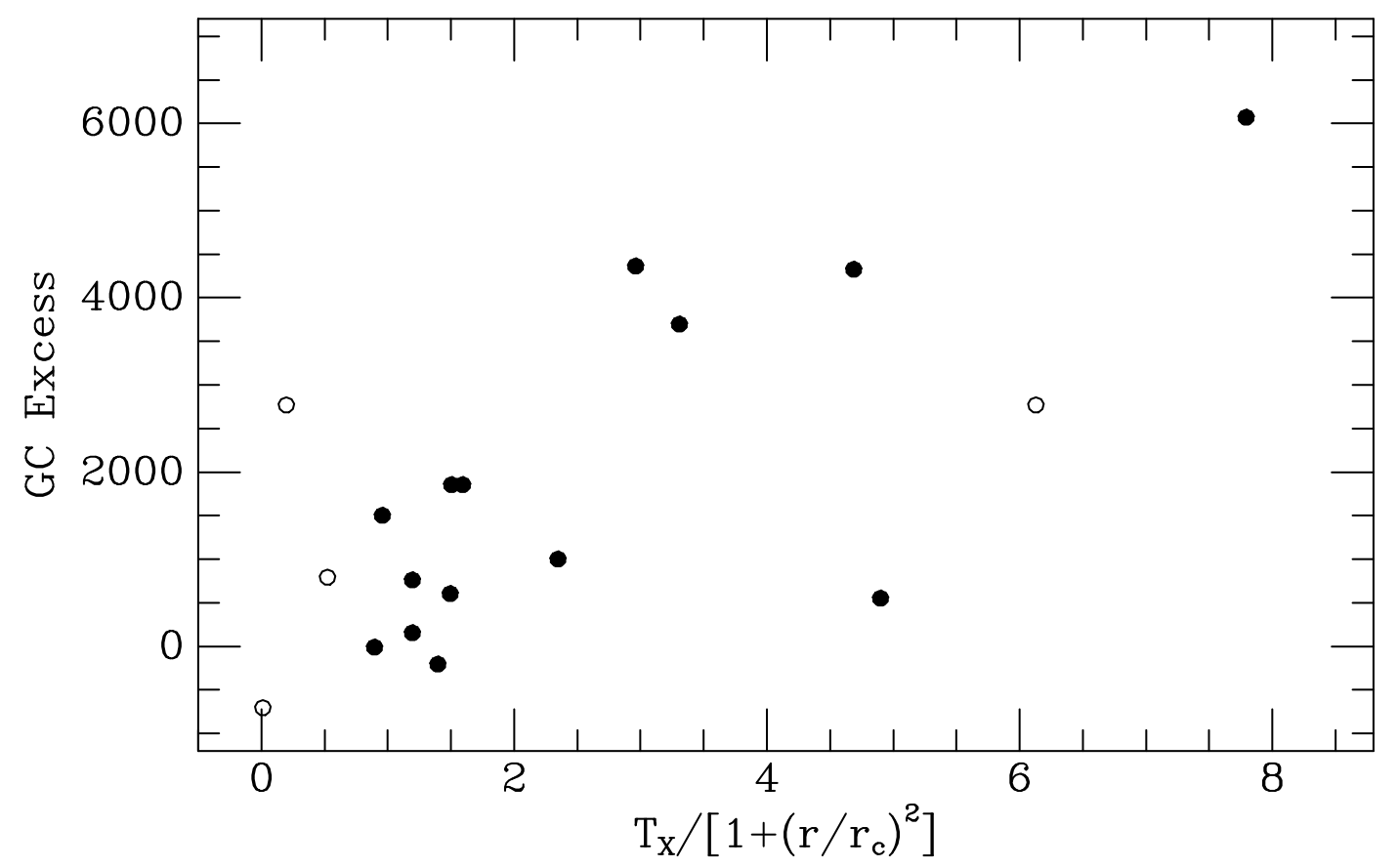

Fig. 13. - The observed "excess" number of GCs in the IGC model is plotted against a quantity proportional to the projected matter density of the cluster at the radial position of the galaxy. (See text for details.) 

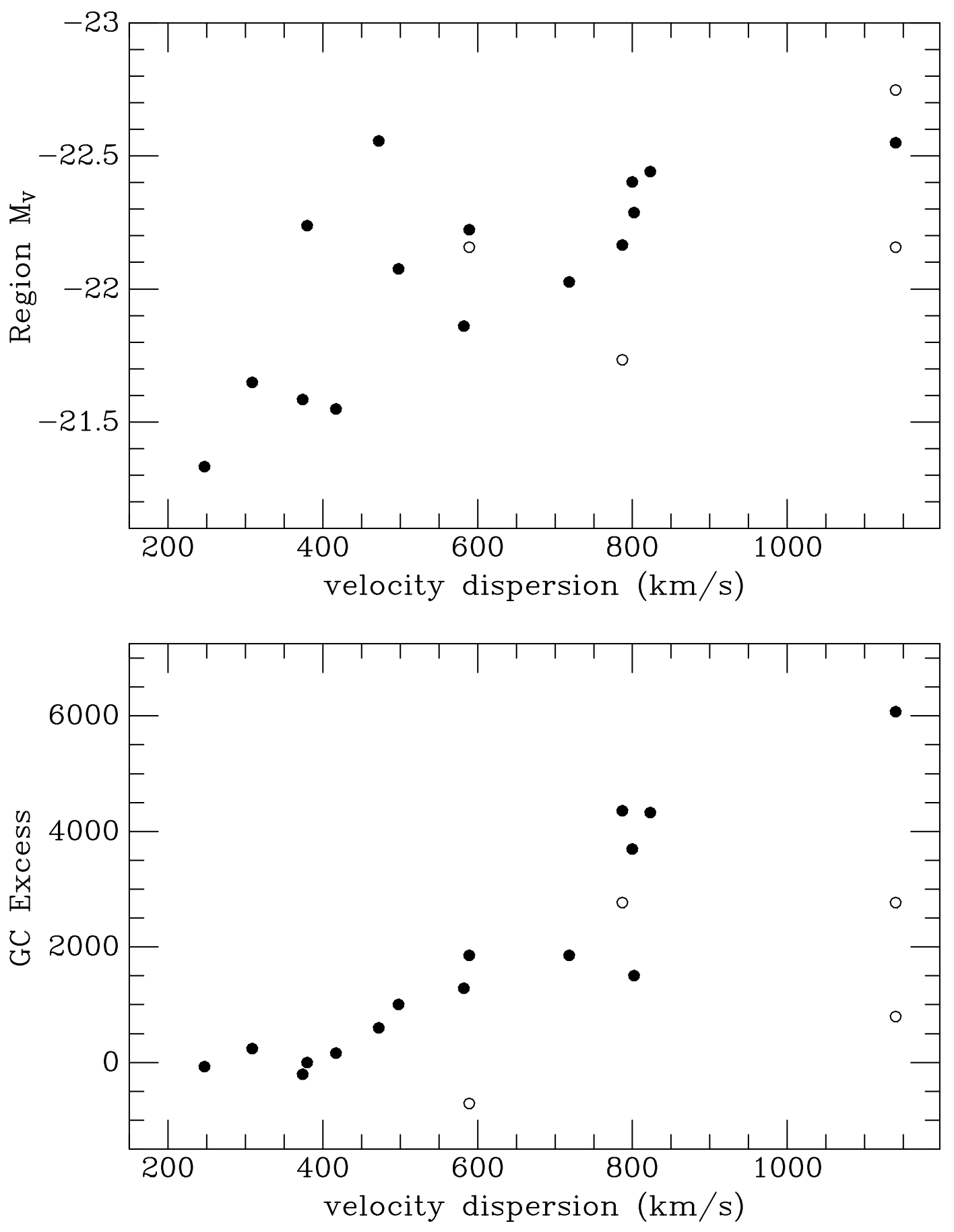

Fig. 14. - A comparison of the sensitivities of galaxy luminosity and GC number to cluster density. In $(a)$, the total absolute $V$ magnitude of the region in each galaxy over which our metric $S_{N}$ values have been meausured (see Figure 5) is plotted against cluster velocity dispersion. In (b), the excess GC number above $S_{N}=3.5$ is plotted against cluster velocity dispersion. 


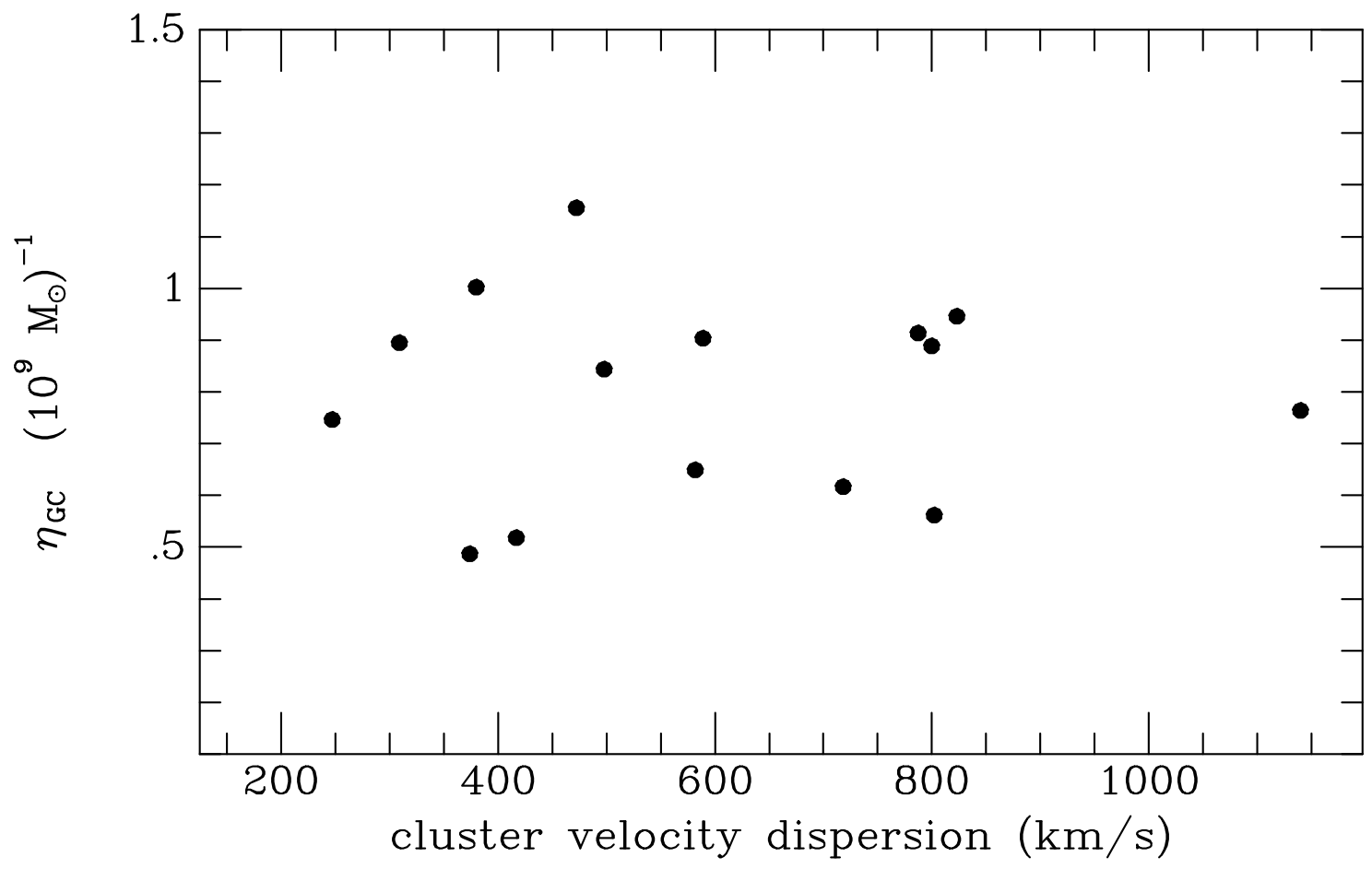

Fig. 15.- The number of GCs per unit $10^{9} M_{\odot}$, denoted $\eta_{G C}$, is plotted against cluster velocity dispersion for the central cluster galaxies in our sample. To estimate the total mass interior to $R=40 \mathrm{kpc}$, we used a cluster core model normalized to the central density of a non-singular isothermal sphere. $\eta_{G C}$ calculated in this way is constant. 